\title{
Pointwise multipliers of Besov spaces of smoothness zero and spaces of continuous functions
}

\section{Herbert Koch and Winfried Sickel}

\begin{abstract}
We characterize the set of pointwise multipliers of the Besov spaces $B_{\infty, 1}^{0}$ and $B_{\infty, \infty}^{0}$. These characterizations are used to obtain regularity results for elliptic partial differential equations. In addition several counterexamples are provided and the relation of various spaces of continuous functions to these multiplier classes are studied.
\end{abstract}

\section{Introduction}

The paper is a first attempt to describe the set of all pointwise multipliers for Besov spaces on the smoothness level 0. We obtain characterizations of multipliers for $B_{\infty, 1}^{0}$ and $B_{\infty, \infty}^{0}$. We call a function $f$ (or distribution) a multiplier for a function space $X$, denoted by $f \in M(X)$, if

$$
\|f \mid M(X)\|=\sup _{h \in X, h \neq 0} \frac{\|f h \mid X\|}{\|h \mid X\|}<\infty .
$$

Since both $f$ and $h$ may be distributions the definition of the product needs some further considerations, which we postpone.

We believe that a study of these multipliers is related to interesting and deep questions in analysis. To support this view we apply our results to elliptic equations.

It has been shown by Frazier and Jawerth in their fundamental paper [5] that $M\left(B_{p q}^{0}\right) \neq L^{\infty}$ unless $p=q=2$. In particular, $B_{2,2}^{0}=L_{2}$ is the only Besov space with $s=0$ where such a description was known before. For

2000 Mathematics Subject Classification: 46E35, 35J15.

Keywords: Besov spaces of smoothness zero, pointwise multipliers, paraproducts characteristic, Dini continuous functions, gradient estimates. 
other Besov spaces than $L^{2}$ with $s=0$ entirely new phenomena occur. The characterization of their multipliers looks quite different than the characterization of spaces of multipliers at different smoothness levels. In view of $B_{\infty, 1}^{0} \hookrightarrow b m o \hookrightarrow B_{\infty, \infty}^{0}$ also the investigations of Janson [12] and Stegenga [27] who characterize $M(b m o)$ are close to ours.

Let $|A|$ be the measure of $A, B(x, r)$ the ball with center $x$ and radius $r, \sup _{|B|<1 / 2}$ the supremum over all balls of volume $1 / 2, f_{B}$ the mean over the ball, $S_{j} f$ is the dyadic truncation in frequency defined in Section 2, cap denotes the capacity, cf. [17] for details, $B_{p q}^{s}$ are the Besov spaces and $F_{p q}^{s}$ the Lizorkin-Triebel spaces defined in Section $2, H_{p}^{s}=F_{p 2}^{s}, H_{p \text {,unif }}^{s}$ a certain uniform variant defined in (2.6) below.

The known multiplier results are essentially the following:

$$
\begin{aligned}
& \left\|f\left|M\left(L_{p}\right)\|=\| f\right| L_{\infty}\right\| \\
& \left\|f\left|M\left(H_{p}^{s}\right)\|\sim\| f\right| H_{p, \text { unif }}^{s}\right\|, \quad s>n / p, \\
& \left\|f\left|M\left(H_{p}^{s}\right)\|\sim\| f\right| L_{\infty}\right\|+\left(\sup _{\substack{A \text { open, } \\
\text { diam } A<1}} \frac{\int_{A}\left(\sum_{j=0}^{\infty}\left|2^{s j} S_{j} f\right|^{2}\right)^{p / 2} d x}{\operatorname{cap}\left(f, H_{p}^{s}\right)}\right)^{1 / p}, s>0, \\
& \left\|f\left|M\left(B_{p, q}^{s}\right)\|\sim\| f\right| B_{p, q, \text { unif }}^{s}\right\|, \quad 1 \leq p \leq q \leq \infty, \quad s>n / p, \\
& \left\|f\left|M\left(B_{\infty, q}^{s}\right)\|\sim\| f\right| B_{\infty, q}^{s}\right\|, \quad s>0, \\
& \left\|f|M(b m o)\|\sim\| f| L_{\infty}\right\|+\sup _{|B| \leq 1 / 2} \frac{|\ln | B||}{|B|} \int_{B}\left|f(x)-f_{B}\right| d x, \\
& \left\|f\left|M\left(B_{\infty, \infty}^{0}\right)\|\sim\| f\right| L_{\infty}\right\|+\left\|f\left|F_{\infty, 1}^{0}\left\|+\sup _{j \geq 0}(1+j)\right\| S_{j} f\right| L_{\infty}\right\|, \\
& \left\|f\left|M\left(B_{\infty, 1}^{0}\right)\|\sim\| f\right| B_{\infty, 1}^{0}\right\| \\
& +\sup _{j \in \mathbb{N}} \sup _{x \in \mathbb{R}^{n}} \sum_{k=0}^{j} \sup _{|y-x| \leq 1} 2^{k n} \int_{B\left(y, 2^{-k}\right)}\left|S_{j} f(z)\right| d z
\end{aligned}
$$

Here the first assertion is trivial. The second represents a famous result of Strichartz [28]. The fourth one in case $p=q$ and $s>n / p$ has been proved by Peetre [20] and for general $s>0$ by Maz'ya and Shaposnikova [17]. Also (1.3) can be found in [17]. This formula generalizes to all spaces of the Lizorkin-Triebel scale which embed into $L^{\infty}$ and their duals, cf. [24]. Formulas (1.4) and (1.5) in the general situation are done in [25]. The multiplier problem is studied as part of a study of function spaces in several monographs, cf. Peetre [20], Triebel [30], Taylor [29] and [21]. The book of 
Maz'ya and Shaposnikova [17] is the only one which is completely devoted to the study of multipliers problems.

Various sufficient conditions for a bounded function $f$ to belong to such a class $M\left(B_{\infty, q}^{0}\right)$ may be derived from the approach via paraproducts, cf. e.g. [21, Chapt. 4], Yamazaki [32], Marschall [14, 15, 16], or Johnsen [13]. However, they do not obtain sufficient and necessary conditions.

The paper at hand deals with (1.7) and (1.8). It provides sharp conditions for $f \in M\left(B_{\infty, 1}^{0}\right)$ and $f \in M\left(B_{\infty, \infty}^{0}\right)$. The characterizations of $M\left(B_{\infty, \infty}^{0}\right)$ and $M\left(B_{\infty, 1}^{0}\right)$ imply for the case of general $p$ and $q(1 \leq p, q \leq \infty)$ that a function $f$ belongs to $M\left(B_{p, q}^{0}\right)$ if

$$
f \in B_{\infty, 1}^{0} \quad \text { and } \quad \sup _{j=1,2, \ldots} j\left\|S_{j} f \mid L_{\infty}\right\|<\infty
$$

(in fact the two conditions in (1.9) characterize those functions which are multipliers for all spaces $B_{p, q}^{0}, 1 \leq p, q \leq \infty$, simultaneously). That follows by duality and complex interpolation. There are however such functions which are not contained in $M(b m o)$.

After deriving the characterization we investigate the relation of $M\left(B_{\infty, \infty}^{0}\right)$, $M(b m o)$ and $M\left(B_{\infty, 1}^{0}\right)$ to classes of continuous functions defined by conditions in terms of moduli of smoothness. Let

$$
\omega(f, r)=\sup _{|x-y|<r}|f(x)-f(y)|, \quad r>0 .
$$

For $\varrho(r)=|\ln r|$ we define

$$
\left\|f\left|C^{\varrho}\|=\| f\right| L_{\infty}\right\|+\sup _{r \leq \frac{1}{2}} \varrho(r) \omega(f, r) .
$$

Recall that $f$ is Dini continuous if

$$
\int_{0}^{1 / 2} \omega(f, r) \frac{d r}{r}<\infty .
$$

Let $C_{D}$ be the space of Dini continuous functions. Then

$$
\begin{gathered}
C_{D} \hookrightarrow C^{\varrho} \hookrightarrow M(b m o) \hookrightarrow M\left(B_{\infty, \infty}^{0}\right), \\
C_{D} \hookrightarrow M\left(B_{\infty, 1}^{0}\right)
\end{gathered}
$$

but

$$
C^{\varrho} \nrightarrow M\left(B_{\infty, 1}^{0}\right) \quad \text { and } \quad M\left(B_{\infty, 1}^{0}\right) \hookrightarrow M\left(B_{\infty, \infty}^{0}\right),
$$

see Lemma 21. 
We have chosen to work with a Fourier-analytic description of the function spaces and not with atoms or wavelets since vanishing moments are not preserved when taking products.

The paper is organized as follows. Section 2 is used to introduce the basic notions including that of the product. It is followed by Section 3 where we collected our main results. In Section 4 we apply the characterization to elliptic problems. Section 5 introduces what we need about paraproducts, which are used in Section 6 to prove the characterizations.

In the remaining part we examine several questions one might ask:

1. Which classes of functions are multipliers resp. are not multipliers (Section 7).

2. Which inclusions do we have among the multiplier spaces (Section 8).

Several properties of Besov and Triebel-Lizorkin spaces are introduced in the appendix where we also investigate relevant subclasses of the space of continuous functions.

\section{Preliminaries}

We denote $a \sim b$ if there exists a constant $c>0$ (independent of the context dependent relevant parameters) such that

$$
c^{-1} a \leq b \leq c a .
$$

By $\mathbb{N}$ we denote the set of natural numbers and by $\mathbb{Z}^{n}$ the set of all lattice points in $\mathbb{R}^{n}$ having integer components. For $\ell \in \mathbb{Z}^{n}$ we define the dyadic cubes

$$
Q_{j, \ell}=\left\{x \in \mathbb{R}^{n}: \quad 2^{-j} \ell_{i} \leq x_{i}<2^{-j}\left(\ell_{i}+1\right), i=1, \ldots, n\right\},
$$

in $\mathbb{R}^{n}$. The symbol $\hookrightarrow$ is used for continuous embedding. Let $\mathcal{S}$ denote the Schwartz class of complex-valued infinitely differentiable and rapidly decreasing functions on $\mathbb{R}^{n}$ and $\mathcal{S}^{\prime}$ its topological dual. As usual, $\mathcal{F}$ denotes the Fourier transform and $\mathcal{F}^{-1}$ its inverse transform, both on $\mathcal{S}^{\prime}$. Let $\varphi_{0} \in \mathcal{S}$ be a radial and real-valued function such that

$$
\varphi_{0}(x) \geq 0, \quad \varphi_{0}(x)=1 \quad \text { if } \quad|x| \leq 1 \quad \text { and } \quad \varphi_{0}(x)=0 \quad \text { if } \quad|x| \geq \frac{3}{2} .
$$

Then, taking

$$
\varphi_{1}(x)=\varphi_{0}\left(\frac{x}{2}\right)-\varphi_{0}(x), \quad \varphi_{j}(x)=\varphi_{1}\left(2^{-j+1} x\right),
$$


for $j=2,3, \ldots$ we obtain a smooth dyadic decomposition of unity:

$$
\sum_{j=0}^{\infty} \varphi_{j}(x)=1, \quad \text { for all } x \in \mathbb{R}^{n} .
$$

We observe that supp $\varphi_{0} \subset \overline{B(0,3 / 2)}$ and $\operatorname{supp} \varphi_{1} \subset \overline{B(0,3)} \backslash B(0,1)$. The dyadic pieces are defined by

$$
S_{j} f(x)=\mathcal{F}^{-1}\left[\varphi_{j}(\xi) \mathcal{F} f(\xi)\right](x), \quad j=0,1, \ldots, \quad f \in \mathcal{S}^{\prime}
$$

and $S^{j}:=\sum_{i=0}^{j} S_{i}$. Let $\psi_{j}=(2 \pi)^{-n / 2} \mathcal{F}^{-1} \varphi_{j}$. Then $S_{j} f=\psi_{j} * f$.

Definition 1 Let $1 \leq p \leq \infty, 1 \leq q \leq \infty$, and $s \in \mathbb{R}$. The Besov space $B_{p, q}^{s}$ is defined to be

$$
B_{p, q}^{s}=\left\{f \in \mathcal{S}^{\prime}: \quad\left\|f \mid B_{p, q}^{s}\right\|=\left(\sum_{j=0}^{\infty} 2^{j s q}\left\|S_{j} f \mid L_{p}\right\|^{q}\right)^{1 / q}<\infty\right\}
$$

with the obvious modifications if $q=\infty$.

Also we need the Triebel-Lizorkin classes with $p=\infty$ for the characterization of the multiplier spaces, cf. (1.7). There are many equivalent norms. The most natural for us is a definition using Carleson measures given by Frazier and Jawerth [5]. The equivalence to other definitions has been shown by Seeger [23].

Definition 2 (i) Let $1 \leq q<\infty$, and $s \in \mathbb{R}$. Then we put

$$
\begin{aligned}
F_{\infty, q}^{s} & =\left\{f \in \mathcal{S}^{\prime}:\right. \\
& \left.\left\|f \mid F_{\infty, q}^{s}\right\|=\sup _{k=0,1, \ldots} \sup _{\ell \in \mathbb{Z}}\left(2^{k n} \int_{Q_{k, \ell}} \sum_{j=k}^{\infty} 2^{j s q}\left|S_{j} f(x)\right|^{q} d x\right)^{1 / q}<\infty\right\}
\end{aligned}
$$

and

$$
F_{\infty, \infty}^{s}=B_{\infty, \infty}^{s} .
$$

The most important space within this scale is $b m o=F_{\infty, 2}^{0}$ which differs from $B M O$ by requiring bounded means for balls of size larger than 1, in contrast to the requirement of bounded mean oscillation for smaller balls, see [7]. Hence the following is an equivalent norm

$$
\left\|f\left|b m o\|=\| S_{0} f\right| L_{\infty}\right\|+\sup _{x, R \leq 1}|B|^{-1} \int_{B(x, R)}\left|f(y)-f_{B}\right| d y .
$$

The norms depend on $\psi$. Different functions $\psi$ lead to equivalent norms. 
Let $\varphi$ be as in (2.2). Then we put

$$
B_{p, q, \text { unif }}^{s}=\left\{f \in \mathcal{S}^{\prime}:\left\|f\left|B_{p, q, u n i f}^{s}\left\|=\sup _{z \in \mathbb{R}^{n}}\right\| \varphi(x-z) f(x)\right| B_{p, q}^{s}\right\|<\infty\right\}
$$

which we equip with the obvious norm.

\section{The definition of the product}

The spaces under consideration here contain singular distributions (at least partly). So the definition of the product needs some care. All functions and distributions will be defined on the Euclidean space $\mathbb{R}^{n}$. If there is no danger of confusion we will omit $\mathbb{R}^{n}$ in the notation. The Schwartz functions are multipliers for all function spaces considered in this paper. Also all function spaces in this paper contain the Schwartz functions. Hence every multiplier of $B_{p q}^{s}$ has to be an element of $B_{p q, u n i f}^{s}$. We require that multipliers lie in $B_{p q, \text { unif }}^{s} \cap B_{p^{\prime}, q^{\prime}, \text { unif }}^{-s}$, which is motivated by the fact that the dual operator should be bounded on the dual space. For $f \in B_{p q}^{s}$ and $g \in B_{p^{\prime}, q^{\prime}, \text { unif }}^{-s}$ we define the product as the distribution

$$
I(\phi)=<\phi g, f>, \quad \phi \in \mathcal{S},
$$

if $B_{p^{\prime}, q^{\prime}}^{-s}=\left(B_{p q}^{s}\right)^{*}$ and with the obvious modifications otherwise. These considerations allow to define the spaces $M\left(B_{p q}^{s}\right)$ in the obvious way. Also the modifications for Lizorkin-Triebel spaces are clear.

We have for all $f \in \mathcal{S}^{\prime}$

$$
\lim _{k \rightarrow \infty} S^{k} f=f \quad\left(\text { convergence in } \mathcal{S}^{\prime}\right) .
$$

In many situations we shall work with these smooth means of the distribution $f$ instead of dealing with the distribution itself. Observe, if either $f \in L_{p}$ or $f$ is uniformly continuous then the convergence in (2.7) takes place in stronger topologies.

There is a second possibility of defining the product:

Definition 3 Let $f, g \in \mathcal{S}^{\prime}$. We define

$$
f \cdot g=\lim _{j \rightarrow \infty} S^{j} f \cdot S^{j} g
$$

whenever the limit on the right-hand side exists in $\mathcal{S}^{\prime}$, where $S^{j}$ is the operator defined in (2.5).

In general, the existence of this limit depends on $\varphi_{0}$, cf. Oberguggenberger [19, Ex. 2.3]. 
If $f$ and $g$ are in $L_{2}^{\text {loc }}$ then the products in both definitions clearly coincide. This is the case for $f \in B_{\infty, 1}^{0}$ and $g \in M\left(B_{\infty, 1}^{0}\right)$ and if $f \in b m o$ and $g \in M(b m o)$. We shall see below that

$$
\left\|g\left|L_{\infty}\|\leq c\| g\right| M\left(B_{\infty, \infty}^{0}\right)\right\|
$$

which again implies uniqueness of the limit for $f \in B_{1,1}^{0} \hookrightarrow L_{1}$, hence by duality uniqueness of the limit for $f \in M\left(B_{\infty, \infty}^{0}\right)$.

\section{Main results}

Now we are in position to formulate the main results of this paper.

Theorem 4 We have

$$
M\left(B_{\infty, \infty}^{0}\right)=F_{\infty, 1}^{0} \cap\left\{f \in L_{\infty}: \sup _{j \in \mathbb{N}}(1+j)\left\|S_{j} f \mid L_{\infty}\right\|<\infty\right\}
$$

and

$$
\left\|f\left|M\left(B_{\infty, \infty}^{0}\right)\|\sim\| f\right| L_{\infty}\right\|+\left\|f\left|F_{\infty, 1}^{0}\left\|+\sup _{j \in \mathbb{N}}(1+j)\right\| S_{j} f\right| L_{\infty}\right\| .
$$

The three conditions appearing in (3.2) are independent of each other, see Lemma 13. There exist discontinuous functions in $M\left(B_{\infty, \infty}^{0}\right)$, cf. Proposition 17. On the other hand the characteristic function of a nontrivial measurable set does never belong to $M\left(B_{\infty, \infty}^{0}\right)$, cf. Proposition 18 .

Theorem 5 The following characterization holds

$$
M\left(B_{\infty, 1}^{0}\right)=\left\{f \in B_{\infty, 1}^{0}: \quad \sup _{j \geq 2, t \in \mathbb{Z}^{n}} \sum_{\ell=0}^{j-2} 2^{\ell n} \max _{Q_{\ell, r} \subset Q_{0, t}} \int_{Q_{\ell, r}}\left|S_{j} f(y)\right| d y<\infty\right\}
$$

$$
\left\|f\left|M\left(B_{\infty, 1}^{0}\right)\|\sim\| f\right| B_{\infty, 1}^{0}\right\|+\sup _{j \geq 2, t \in \mathbb{Z}^{n}} \sum_{\ell=0}^{j-2} 2^{\ell n} \max _{Q_{\ell, r} \subset Q_{0, t}} \int_{Q_{\ell, r}}\left|S_{j} f(y)\right| d y .
$$

Again the two conditions in (3.3) are independent of each other, cf. Lemma 16. 


\section{Elliptic estimates}

Let $\Omega \subset \mathbb{R}^{n}$ be a bounded and open set. We denote by $d(x)$ the distance to the boundary. Once and for all we choose a nonnegative radial function $\eta \in C_{0}^{\infty}$ supported in the ball $B(0,3 / 2)$, with $\left.\eta\right|_{B(0,1)}=1$. Let $A$ be one of the spaces under consideration here. We define

$$
\left\|f\left|A(\Omega)\left\|=\sup _{y \in \Omega} d(y)\right\| \eta\left(4 \frac{(\cdot-y)}{d(y)}\right) f\right| A\right\| .
$$

It is important for using this definition that smooth functions with compact support are multipliers in $B_{p, q}^{s}$ and $F_{p, q}^{s}$.

We define the Riesz transform by

$$
\mathcal{F}\left[R_{j} f\right](\xi)=\frac{\xi_{j}}{|\xi|} \mathcal{F} f(\xi), \quad j=1, \ldots, n,
$$

for all Schwartz functions $f$. Then

$$
R_{i} R_{j} \Delta f=\partial_{i} \partial_{j} f .
$$

The mapping $f \mapsto \eta R_{i} R_{j}(\eta f)$ extends to a bounded mapping of all TriebelLizorkin and Besov spaces into itself. Here we need a slightly more restricted version: we may and do assume that $s=0$.

Suppose that $f$ is supported in a ball $B(0,1)$. If $n \geq 3$ there is a unique distribution $u$, which is continuous for large $x$ and which decays to zero as $x \rightarrow \infty$, which satisfies

$$
\Delta u=f
$$

Then

$$
|\nabla u(x)| \leq c(1+|x|)^{1-n}, \quad\left|D_{x}^{2} u(x)\right| \leq c(1+|x|)^{-n}
$$

for $|x| \geq 2$,

$$
\left|S_{j} \nabla u(x)\right| \leq c_{N}(1+|x|)^{-N}
$$

for all $N$ if $j \geq 1$ and $|x| \geq 2$ and

$$
\left|S_{0} \nabla u(x)\right| \leq c(1+|x|)^{1-n} .
$$

There is only a marginal difference for $n=2$; there is (up to the addition of constants) a unique solution whose derivative decays at infinity. In particular, by similar arguments, if

$$
\Delta u=\nabla \cdot f,
$$

if $f \in\left(\mathcal{S}^{\prime}\right)^{n}$ is supported in the unit ball and if $u$ satisfies some mild restriction at infinity (at least if $p>1$ ), then

$$
\|\nabla u|A\|\leq c\| f| A\| \text {. }
$$


We shall use a slightly different version, which follows by the same arguments:

$$
\left\|\eta R_{i} R_{j} f|A\|\leq c\| f| A\right\|
$$

for $f$ supported in the unit ball.

In the sequel we shall need estimates with a loss of one derivative. It is crucial that in this case the choice of the function space is much less important.

Clearly, if $\Delta u=f$ and $f$ is supported in the unit ball,

$$
\|\eta \nabla u|A\|\leq c\| f| A\| .
$$

This estimate can be improved for small balls. Let $r \leq 1, \eta_{r}(x)=\eta(r x)$ and $u_{r}(x)=u(r x)$. We obtain

$$
\begin{aligned}
\left\|\eta_{4 / r} \nabla u \mid A\right\| & \leq c\left\|\eta_{4 / r} \nabla u \mid B_{p 1}^{0}\right\| \\
& \leq c r^{-1+\frac{n}{p}}\left\|\eta \nabla u_{r / 4} \mid B_{p 1}^{0}\right\| \\
& \leq c r^{-1+\frac{n}{p}}\left\|\eta_{2} D_{x}^{2} u_{r / 4} \mid B_{p \infty}^{0}\right\| \\
& \leq c r\left\|\eta_{2 / r} D_{x}^{2} u \mid B_{p \infty}^{0}\right\| \\
& \leq c r\left\|\eta D_{x}^{2} u \mid B_{p \infty}^{0}\right\| \\
& \leq c r\left\|f \mid B_{p \infty}^{0}\right\| \\
& \leq c r\|f \mid A\| .
\end{aligned}
$$

We used the embedding $B_{p, q}^{0} \hookrightarrow B_{p, 1}^{0}$ for the first inequality, obvious scaling and

$$
\left\|S^{j} f\left|L_{p}\left\|\leq \sum_{i=0}^{j}\right\| S_{i} f\right| L_{p}\right\|
$$

for the second inequality, the Poincaré type inequality $\left\|v\left|B_{p, 1}^{0}\|\leq c\| \nabla v\right| B_{p, \infty}^{0}\right\|$ for functions supported in the ball of radius 3 for the third inequality, the fact that smooth compactly supported functions are multipliers for the fourth, scaling for the fifth, bounds for the Riesz transforms for the sixth, and obvious embeddings for the last inequality. It is clear that we may replace $f$ by $\eta_{(r / 2)^{-1}} f$ on the right hand side.

The Poincaré inequality can be somewhat sharpened by the same arguments, but using the Ehrling lemma:

$$
\begin{aligned}
\left\|\left(\nabla \eta_{4 / r}\right) u \mid A\right\| & \leq c\left\|\left(\nabla \eta_{4 / r}\right) u \mid B_{p, 1}^{0}\right\| \\
& \leq c r^{-1+\frac{n}{p}}\left\|(\nabla \eta) u_{r / 4} \mid B_{p 1}^{0}\right\| \\
& \leq r^{-1+\frac{n}{p}} c(\varepsilon)\left\|\eta_{2} u_{r / 4}\left|L_{p}\left\|+\varepsilon r^{-1+\frac{n}{p}}\right\| \eta_{2} \nabla u_{r / 4}\right| B_{p \infty}^{0}\right\| \\
& \leq r^{-1} c(\varepsilon)\left\|\eta_{2 / r} u\left|L_{p}\|+\varepsilon\| \eta_{2 / r} \nabla u\right| A\right\| .
\end{aligned}
$$


Let $a^{i j}$ be measurable functions.

Theorem 6 Let $A=B_{p q}^{0}$ or $A=F_{p q}^{0}$ with $1 \leq p, q \leq \infty$. Then there exists $\delta>0$ such that

$$
\left\|a^{i j}-\delta^{i j} \mid M(A)\right\| \leq \delta
$$

$\nabla u \in A^{l o c}$ and

$$
\partial_{i}\left(a^{i j} \partial_{j} u\right)=\partial_{i} f^{i} \quad \text { in } \Omega
$$

imply

$$
\|\nabla u|A(\Omega)\|\leq c\| f| A(\Omega)\|+c\left\|u \mid L_{p}\right\| .
$$

Proof. It suffices to prove an apriori estimate. Then, with $v=\eta_{4 / r} u$, and omitting the index of $\eta$

$$
\partial_{i}\left(a^{i j} \partial_{j} v\right)=\partial_{i}\left(a^{i j}\left(\partial_{j} \eta\right) u\right)+\left(\partial_{i} \eta\right) a^{i j} \partial_{j} u+\left(\partial_{i} f^{i}\right) \eta
$$

hence

$$
\begin{aligned}
\Delta v= & \partial_{i}\left[\left(\delta^{i j}-a^{i j}\right) \partial_{j} v\right]+\partial_{i}\left(a^{i j}\left(\partial_{j} \eta\right) u\right)+\left(\partial_{i} \eta\right)\left(a^{i j}-\delta^{i j}\right) \partial_{j} u \\
& +\partial_{i}\left(u \partial_{i} \eta\right)-u \Delta \eta+\partial_{i}\left(\eta f^{i}\right)+\left(\partial_{i} \eta\right) f^{i} .
\end{aligned}
$$

and hence, by (4.2), the assumption we have

$$
\begin{aligned}
\|\nabla v \mid A\| \leq & c\left(\delta\|\nabla u|A\|+\| u \nabla \eta| A\|+r\left\|u \Delta \eta \mid L_{p}\right\|\right. \\
& \left.+\left\|\eta_{r^{-1}} f|A\|+d\| f \nabla \eta| B_{p \infty}^{0}\right\|\right) \\
\leq & c \delta r^{-1}\|\nabla v \mid A(\Omega)\|+c r^{-1}\left(\left\|u\left|L_{p}\|+\| f\right| A(\Omega)\right\|\right) .
\end{aligned}
$$

We complete the proof by taking the supremum with respect to $x$, choose $\delta$ small and subtract the first term of the right hand side from both sides.

Corollary 7 Suppose that

$$
\left\|a^{i j}-\delta^{i j} \mid M\left(B_{\infty, 1}^{0}\right)\right\| \leq \delta
$$

$u \in L_{\infty}, \nabla u \in B_{\infty, 1}^{0, l o c}(\Omega)$ and $\partial_{i}\left(a^{i j} \partial_{j} u\right)=0$ in $\Omega \subset \mathbb{R}^{n}$. Then

$$
\sup d(x)|\nabla u(x)| \leq c\left\|u \mid L_{\infty}\right\| .
$$

Proof. This is an immediate consequence of the previous result applied with $A=B_{\infty, 1}^{0}$ since $B_{\infty, 1}^{0} \hookrightarrow L_{\infty}$.

Remark 8 This has been proven (for $f \equiv 0$ ) by Grüter and Widman [11] by completely different methods assuming Dinicontinuity of the coefficients. Still other methods have been used by Cafarelli and Kenig [3] for parabolic problems. They require a local Dini condition. Our conditions ensuring $u \in C^{1}$ are slightly weaker than Dini continuity for the coefficients: $C_{D} \hookrightarrow$ $M\left(B_{\infty, 1}^{0}\right)$ (see Lemma 20) and, for $f \in C_{D}$ this norm becomes small if one considers only small balls. It may be of independent interest that the bound on the gradient is obtained by a perturbation argument. 


\section{Paraproducts and properties of $g \mapsto f g$}

It is the purpose of the first subsection to clarify that several possibilities for defining $M(A)$ yield the same for the spaces considered here. The second subsection provides tools which we shall use in the proof of the main results.

\subsection{Some elementary properties of the operator $g \mapsto f g$}

We start with some notation. The operator $g \mapsto f g$ will be denoted by $T_{f}$. Further, we put $\mathcal{L}\left(B_{p, q}^{s}\right)$ the Banach space of bounded linear maps form $B_{p, q}^{s}$ to itself with the obvious norm and

$$
\tilde{M}\left(B_{p, q}^{s}\right)=\left\{f \in \mathcal{S}^{\prime}: \quad f g \in B_{p, q}^{s} \quad \text { for all } \quad g \in B_{p, q}^{s}\right\} .
$$

For a moment we shall be a bit more general than needed later on.

Lemma 9 Suppose $1 \leq p, q \leq \infty$ and $s \in \mathbb{R}$. Then $f \in \tilde{M}\left(B_{p, q}^{s}\right)$ implies $T_{f} \in \mathcal{L}\left(B_{p, q}^{s}\right)$.

This may be proven either by an application of the uniform boundedness principle to $g \rightarrow S^{j}(f g)$ or by the closed graph theorem as in Maz'ya and Shaposnikova [17].

In what follows we interpret $\tilde{M}\left(B_{p, q}^{s}\right)$ as a subspace of $\mathcal{L}\left(B_{p, q}^{s}\right)$, that means we identify $f$ with the corresponding operator $T_{f}$. In other words, we identify $M$ and $\tilde{M}$ and drop the tilde in the sequel. We continue with some well-known assertions, cf. e.g. [21, 4.3.2, 4.6.3, 4.9].

Lemma 10 Suppose $1 \leq p, q \leq \infty$ and $s \in \mathbb{R}$.

(i) It holds $M\left(B_{p, q}^{s}\right)=M\left(B_{p^{\prime}, q^{\prime}}^{-s}\right)$ and

$$
\left\|f\left|M\left(B_{p, q}^{s}\right)\|\leq c\| f\right| M\left(B_{p^{\prime}, q^{\prime}}^{-s}\right)\right\| .
$$

(ii) We have $M\left(B_{p, q}^{s}\right) \hookrightarrow L_{\infty}$ and

$$
\left\|f\left|L_{\infty}\|\leq c\| f\right| M\left(B_{p, q}^{s}\right)\right\|
$$

(iii) It holds $M\left(B_{p, q}^{s}\right) \hookrightarrow B_{p, q, \text { unif }}^{s}$.

(iv) Let $\varphi \in L_{1}$. If $f \in M\left(B_{p, q}^{s}\right)$, then $\varphi * f \in M\left(B_{p, q}^{s}\right)$ and

$$
\left\|\varphi * f\left|M\left(B_{p, q}^{s}\right)\|\leq\| \varphi\right| L_{1}\right\|\left\|f \mid M\left(B_{p, q}^{s}\right)\right\| .
$$


Later on we need also the following

Lemma 11 Suppose $1 \leq p, q \leq \infty$ and $s \in \mathbb{R}$. Then

$$
\left\|T_{f}\left|\mathcal{L}\left(B_{p, q}^{s}\right)\left\|\sim \limsup _{j \rightarrow \infty}\right\| T_{S^{j} f}\right| \mathcal{L}\left(B_{p, q}^{s}\right)\right\|
$$

holds for all $f \in M\left(B_{p, q}^{s}\right)$.

Proof. The inequality

$$
\left\|T_{S^{j} f}\left|\mathcal{L}\left(B_{p, q}^{s}\right)\|\leq\| T_{f}\right| \mathcal{L}\left(B_{p, q}^{s}\right)\right.
$$

is a consequence of Lemma 10(iv). Concerning the reverse inequality we employ the Fatou property of the underlying distribution spaces. If $f \in$ $M\left(B_{p, q}^{s}\right)$, then by definition $\lim _{j \rightarrow \infty} S^{j} f S^{j} g=f g$ (convergence in $\mathcal{S}^{\prime}$ ) and by Lemma 10(iv)

$$
\sup _{j=0,1, \ldots}\left\|S^{j} f S^{j} g\left|B_{p, q}^{s}\|\leq c\| T_{f}\right| \mathcal{L}\left(B_{p, q}^{s}\right)\right\|\left\|g \mid B_{p, q}^{s}\right\| .
$$

Now the Fatou property of $B_{p, q}^{s}$, cf. e.g. Franke [4] or Bourdaud and Meyer [2], implies

$$
\left\|f g\left|B_{p, q}^{s}\left\|\leq C \liminf _{j \rightarrow \infty}\right\| S^{j} f S^{j} g\right| B_{p, q}^{s}\right\|
$$

for some $C$ independent of $f$ and $g$.

\subsection{Paraproducts}

Let $\varphi_{0}$ be as in (2.2) and $\left\{\varphi_{j}\right\}_{j=0}^{\infty}$ a corresponding decomposition of unity.

We have

$$
\begin{aligned}
& (f \cdot g)(x)=\lim _{j \rightarrow \infty} S^{j} f(x) S^{j} g(x) \\
& \quad=\sum_{k=2}^{\infty} \sum_{\ell=0}^{k-2} S_{\ell} f(x) S_{k} g(x)+\sum_{k=0}^{\infty} \sum_{\ell=k-1}^{k+1} S_{\ell} f(x) S_{k} g(x)+\sum_{\ell=2}^{\infty} \sum_{k=0}^{\ell-2} S_{\ell} f(x) S_{k} g(x)
\end{aligned}
$$

(here we put $S_{-1} f \equiv 0$ ), whenever the three sums on the right-hand side make sense in $\mathcal{S}^{\prime}$. Observing $\sum_{\ell=0}^{k-2} S_{\ell} f=S^{k-2} f$ we may rewrite these sums as

$$
\begin{aligned}
& \Pi_{1}(f, g)(x)=\sum_{k=2}^{\infty} S^{k-2} f(x) S_{k} g(x), \\
& \Pi_{2}(f, g)(x)=\sum_{k=0}^{\infty} \sum_{\ell=k-1}^{k+1} S_{\ell} f(x) S_{k} g(x), \\
& \Pi_{3}(f, g)(x)=\sum_{\ell=2}^{\infty} S_{\ell} f(x) S^{\ell-2} g(x) .
\end{aligned}
$$


The bilinear operators $\Pi_{i}, i=1,3$ are called paraproducts. Their usefulness comes to a large extent from the observation that the Fourier transforms of $S^{k-2} f S_{k} g$ and $S_{k} f S^{k-2} g$ are supported in $\left\{\xi: 2^{k-2} \leq|\xi| \leq 2^{k+2}\right\}$. Thanks to Lemma 10(ii) we know

$$
\left\|\Pi_{1}(f, g)\left|B_{p, q}^{s}\|\leq c\| f\right| L_{\infty}\right\|\left\|g \mid B_{p, q}^{s}\right\|
$$

where $c$ does not depend on $f$ and $g$. Hence, in case we deal with sufficient conditions it remains to estimate the paraproducts $\Pi_{2}$ and $\Pi_{3}$.

\section{The pointwise multipliers of $B_{\infty, \infty}^{0}$ and $B_{\infty, 1}^{0}$}

In the first part of this section we shall give the proof of Theorem 4 . The proof of Theorem 5 is given in the second part.

\subsection{The characterization of $M\left(B_{\infty, \infty}^{0}\right)$ - Proof of Theorem 4}

Proof. Step 1. Sufficiency. As pointed out in Subsection 2.3, cf. (5.4), it will be sufficient to estimate $\Pi_{2}$ and $\Pi_{3}$.

Substep 1.1. Estimate of $\Pi_{2}$. Inspecting the supports of the Fourier transforms one obtains the identity

$$
S_{k}\left(\Pi_{2}(f, g)\right)=\sum_{j=k-3}^{\infty} \sum_{\ell=-1}^{1} S_{k}\left(S_{j+\ell} f S_{j} g\right), \quad k=3,4, \ldots
$$

Observe further, that for each natural number $M$ there exists a constant $c_{M}$ such that

$$
\left|\psi_{k}(x)\right|=(2 \pi)^{-n / 2}\left|\mathcal{F}^{-1} \varphi_{k}(x)\right| \leq c_{M} 2^{k n}\left(1+2^{k}|x|\right)^{-M}
$$

holds for all $x \in \mathbb{R}^{n}$, cf. (2.3). Concentrating on $k \geq 3$ we find

$$
\begin{aligned}
\left|S_{k}\left(\Pi_{2}(f, g)\right)(0)\right| & \leq C \sum_{\ell=-1}^{1} \sum_{m \in \mathbb{Z}^{n}} \int_{Q_{k-3, m}} 2^{k n}\left(1+2^{k}|y|\right)^{-M} \sum_{j=k-3}^{\infty}\left|S_{j+\ell} f(y) S_{j} g(y)\right| d y \\
& \leq C\left\|f\left|F_{\infty, 1}^{0}\left\|\sup _{j=0,1, \ldots}\right\| S_{j} g\right| L_{\infty}\right\| \sum_{m \in \mathbb{Z}^{n}}(1+|m|)^{-M} \\
& \leq C\left\|f\left|F_{\infty, 1}^{0}\|\| g\right| B_{\infty, \infty}^{0}\right\|,
\end{aligned}
$$

where $C$ does not depend on $f$ and $g$. A simple shift argument yields the same estimate for all $x \in \mathbb{R}^{n}$. This gives

$$
\left\|\Pi_{2}(f, g)\left|B_{\infty, \infty}^{0}\|\leq c\| f\right| F_{\infty, 1}^{0}\right\|\left\|g \mid B_{\infty, \infty}^{0}\right\| .
$$


Substep 1.2. The Fourier transform $S_{j} f S^{j-2} g$ is supported in $A=\{\xi$ : $\left.2^{j-1}|\xi| \leq 2^{j+2}\right\}$. Hence

$$
S_{j} \Pi_{3}(f, g)=\sum_{i=-2}^{1} S_{j}\left(S_{j+i} f S^{j+i-2} g\right)
$$

and

$$
\begin{aligned}
\left\|\Pi_{3}(f, g) \mid B_{\infty, \infty}^{0}\right\| & \leq c \sup _{j=2,3, \ldots}\left\|S_{j} f S^{j-2} g \mid L_{\infty}\right\| \\
& \leq c\left(\sup _{j=2,3, \ldots} j\left\|S_{j} f \mid L_{\infty}\right\|\right) \sup _{j=2,3, \ldots} \frac{1}{j} \sum_{\ell=0}^{j-2}\left\|S_{\ell} g \mid L_{\infty}\right\| \\
& \leq c \sup _{j=2,3, \ldots} j\left\|S_{j} f\left|L_{\infty}\|\| g\right| B_{\infty, \infty}^{0}\right\|
\end{aligned}
$$

where $c$ does not depend on $f$ and $g$.

Step 2. Necessity follows from the following result and Lemma 10(ii).

Proposition 12 Suppose $f \in M\left(B_{\infty, \infty}^{0}\right)$. Then

$$
\sup _{j=0,1, \ldots}(1+j)\left\|S_{j} f\left|L_{\infty}\|+\| f\right| F_{\infty, 1}^{0}\right\| \leq c\left\|f \mid M\left(B_{\infty, \infty}^{0}\right)\right\| .
$$

Proof. Suppose that $f \in M\left(B_{\infty, \infty}^{0}\right)$. Then

$$
\left\|S_{j} f\left|M\left(B_{\infty, \infty}^{0}\right)\|\leq c\| f\right| M\left(B_{\infty, \infty}^{0}\right)\right\|
$$

by Lemma 10. Now we test $T_{S_{j} f}$ with $g(x)=\sum_{\ell=0}^{j-2} e^{i 2^{\ell} x_{1}} \in B_{\infty, \infty}^{0}$ which depends on $j$, but with a uniformly bounded norm. Obviously, because of the support of the Fourier transform,

$$
\left\|\Pi_{1}\left(S_{j} f, g\right)\left|B_{\infty, \infty}^{0}\|\leq c\| f\right| L_{\infty}\right\|\left\|g \mid B_{\infty, \infty}^{0}\right\|
$$

and

$$
\begin{aligned}
\left\|\Pi_{2}\left(S_{j} f, g\right) \mid B_{\infty, \infty}^{0}\right\| & \leq c \sup _{j=0,1, \ldots} \sup _{\ell=-1,0,1}\left\|\left(S_{j+\ell} f\right) e^{i 2^{j} x_{1}} \mid L_{\infty}\right\| \\
& \leq C\left\|f\left|L_{\infty}\|\| g\right| B_{\infty, \infty}^{0}\right\| .
\end{aligned}
$$

Hence, $S_{j} f \cdot g$ is an uniformly bounded sequence in $B_{\infty, \infty}^{0}$ if and only if $\Pi_{3}\left(S_{j} f, g\right)$ is such a sequence. We choose $x^{j}$ with $\sup _{x}\left|S_{j} f(x)\right| \leq 2\left|S_{j} f\left(x^{j}\right)\right|$ and replace $g$ by $g_{j}(x)=g\left(x-x^{j}\right)$. Then the above arguments can be applied 
as well for $S_{j} f \cdot g_{j}$ instead of $S_{j} f \cdot g$. If $j \geq 2$ we find (since by the support of the Fourier transforms $\left.\left(S_{j-1}+S_{j}+S_{j+1}\right) \Pi_{3}\left(S_{j} f, g_{j}\right)=\Pi_{3}\left(S_{j} f, g_{j}\right)\right)$

$$
\begin{aligned}
\left\|\Pi_{3}\left(S_{j} f, g_{j}\right) \mid B_{\infty, \infty}^{0}\right\| & =\sup _{k}\left\|S_{k}\left(\sum_{\ell=0}^{j-2} e^{i 2^{\ell}\left(x_{1}-x_{1}^{j}\right)} S_{j} f\right) \mid L_{\infty}\right\| \\
& \geq \frac{1}{3 c}\left\|\sum_{\ell=0}^{j-2} e^{i 2^{\ell}\left(x_{1}-x_{1}^{j}\right)} S_{j} f \mid L_{\infty}\right\| \\
& \geq \frac{j-1}{3 c}\left|S_{j} f\left(x^{j}\right)\right|
\end{aligned}
$$

where $c=\left\|\psi_{1} \mid L_{1}\right\|$. Hence, from the uniform boundedness of $T_{S_{j} f}\left(g_{j}\right)$ in $B_{\infty, \infty}^{0}$ the estimate

$$
c \sup j\left\|S_{j} f\left|L_{\infty}\|\geq\| f\right| M\left(B_{\infty, \infty}^{0}\right)\right\|
$$

follows.

We recall that $\psi_{1}$ is a real and radial Schwartz function. Further, we fix $\delta>0$ small and choose $\varrho \in C_{0}^{\infty}$ such that

$$
\text { supp } \varrho \subset\left\{x: \psi_{1}(x) \geq \delta\right\}
$$

and put $\varrho_{k}(x)=\varrho\left(2^{k} x\right)$. Finally, let

$$
g_{k}(x)=\sum_{j=k+N}^{\infty} S_{j}\left(\varrho_{k} \frac{S_{j} f}{S_{j}^{*} f}\right)(x), \quad k=0,1, \ldots,
$$

where $N$ will be chosen later and

$$
S_{j}^{*} f(x)=\sup _{y \in \mathbb{R}^{n}} \frac{\left|S_{j} f(x-y)\right|}{\left(1+2^{j}|y|\right)^{2 n}}, \quad x \in \mathbb{R}^{n}
$$

is a maximal function of Peetre-Fefferman-Stein type.

By definition $\left|S_{j} f(x)\right| \leq S_{j}^{*} f(x)$. Moreover, as it is obvious from the definition, either $S_{j}^{*} f \equiv 0$ (if and only if $S_{j} f \equiv 0$ ) or it never vanishes. So the quotient makes sense and defines a function in $L_{\infty}$ with norm $\leq 1$. We claim that $g_{k}, k=0,1,2, \ldots$ is a uniformly bounded family in $B_{\infty, \infty}^{0}$. To see this observe

$$
\left\|S_{\ell} g_{k}\left|L_{\infty}\left\|\leq c \sup _{|\ell-j| \leq 1}\right\| S_{j}\left(\varrho_{k} \frac{S_{j} f}{S_{j}^{*} f}\right)\right| L_{\infty}\right\| \leq c\|\rho\|_{\infty} .
$$


Hence we have

$$
\begin{aligned}
\left\|f g_{k} \mid B_{\infty, \infty}^{0}\right\| & \geq\left|S_{k}\left(f g_{k}\right)(0)\right| \\
& =\left|2^{-k n} \sum_{j=k+N}^{\infty} \int_{\mathbb{R}^{n}} \psi_{k}(x) f(x) S_{j}\left(\varrho_{k} \frac{S_{j} f}{S_{j}^{*} f}\right)(x) d x\right| \\
& \geq\left|2^{-k n} \sum_{j=k+N}^{\infty} \int_{\mathbb{R}^{n}} S_{j}\left(\psi_{k} f\right)(y) \varrho_{k}(y) \frac{S_{j} f(y)}{S_{j}^{*} f(y)} d y\right| .
\end{aligned}
$$

Our next aim consists in replacing $S_{j}\left(f \psi_{k}\right)(y)$ by $S_{j} f(y) \psi_{k}(y)$. To justify this we use the following commutator estimate

$$
\begin{aligned}
& \left|\int S_{j}\left(f \psi_{k}\right)(y) \varrho\left(2^{k} y\right) \frac{S_{j} f(y)}{S_{j}^{*} f(y)} d y-\int S_{j} f(y) \psi_{k}(y) \varrho\left(2^{k} y\right) \frac{S_{j} f(y)}{S_{j}^{*} f(y)} d y\right| \\
& =\left|\iint \psi_{j}(y-z) f(z)\left[\psi_{k}(z)-\psi_{k}(y)\right] d z \varrho\left(2^{k} y\right) \frac{S_{j} f(y)}{S_{j}^{*} f(y)} d y\right| \\
& \quad \leq c 2^{j n} \int_{\text {supp } \varrho_{k}} \int \frac{\left|\psi_{k}(z)-\psi_{k}(y)\right|}{\left(1+2^{j}|y-z|\right)^{M}} d z d y\left\|f \left|L_{\infty}\left\||| \frac{S_{j} f}{S_{j}^{*} f} \mid L_{\infty}\right\|\right.\right. \\
& \leq C_{1} 2^{k-j}\left\|f \mid L_{\infty}\right\|
\end{aligned}
$$

with $C_{1}$ independent of $f, g, j$ and $k$. Now

$$
\begin{aligned}
& \left|\sum_{j=k+N}^{\infty} \int S_{j} f(y) \psi_{k}(y) \varrho\left(2^{k} y\right) \frac{S_{j} f(y)}{S_{j}^{*} f(y)} d y\right| \\
& =\left|\sum_{j=k+N}^{\infty} \int \varrho\left(2^{k} y\right) \psi_{k}(y) \frac{\left[S_{j} f(y)\right]^{2}}{S_{j}^{*} f(y)} d y\right| \geq \delta 2^{k n} \int_{\text {supp } \varrho_{k}} \sum_{j=k+N}^{\infty} \frac{\left[S_{j} f\right]^{2}(y)}{S_{j}^{*} f(y)} d y,
\end{aligned}
$$

where we have used the specific relation between $\varrho$ and $\psi$. Replacing $\varrho_{k} \psi_{k}$ by $\varrho_{k}\left(x-x^{k, \ell}\right) \psi_{k}\left(x-x^{k, \ell}\right)$, where $x^{k, \ell}$ denotes the center of $Q_{k, \ell}$ we may use the same arguments as before without changing even the constants in the inequalities. Moreover, we may extend the integration over $Q_{k, \ell}$ instead of supp $\varrho_{k}\left(\cdot-x^{k, \ell}\right)$ by using a fixed finite number of shifted copies of $\varrho_{k} \psi_{k}$. Summing up over $j$ in (6.5) to control the commutator terms we obtain

$$
\left\|f\left|M\left(B_{\infty, \infty}^{0}\right)\left\|\geq C_{2} \sup _{k \in \mathbb{N}, \ell \in \mathbb{Z}^{n}} 2^{k n} \int_{Q_{k, \ell}} \sum_{j=k+N}^{\infty} \frac{\left[S_{j} f\right]^{2}(y)}{S_{j}^{*} f(y)} d y-C_{1} 2^{-N}\right\| f\right| L_{\infty}\right\|,
$$

where $C_{1}$ and $C_{2}$ are positive constants independent of $f$ and $N \geq 3$. Taking into account Lemma 10 (ii) we arrive at

$$
\left\|f \mid M\left(B_{\infty, \infty}^{0}\right)\right\| \geq C \sup _{k \in \mathbb{N}, \ell \in \mathbb{Z}^{n}} 2^{k n} \int_{Q_{k, \ell}} \sum_{j=k+N}^{\infty} \frac{\left[S_{j} f\right]^{2}(y)}{S_{j}^{*} f(y)} d y .
$$


It remains to compare

$$
\int_{Q_{k, \ell}} \frac{\left[S_{j} f\right]^{2}(y)}{S_{j}^{*} f(y)} d y \quad \text { and } \quad \int_{Q_{k, \ell}}\left|S_{j} f(y)\right| d y .
$$

By Hölder's inequality

$$
\begin{aligned}
\int_{Q_{k, \ell}}\left|S_{j} f(y)\right| d y & =\int_{Q_{k, \ell}}\left|S_{j} f(y)\right|\left[\frac{S_{j}^{*} f(y)}{S_{j}^{*} f(y)}\right]^{1 / 2} d y \\
& \leq\left(\int_{Q_{k, \ell}} S_{j}^{*} f(y) d y\right)^{1 / 2}\left(\int_{Q_{k, \ell}} \frac{\left|S_{j} f(y)\right|^{2}}{S_{j}^{*} f(y)} d y\right)^{1 / 2} .
\end{aligned}
$$

A maximal inequality proved by Ryshkov [22] shows that

$$
\sup _{\ell \in \mathbb{Z}^{n}} \int_{Q_{k, \ell}}\left|S_{j}^{*} f(y)\right| d y \leq c \sup _{\ell \in \mathbb{Z}^{n}} \int_{Q_{k, \ell}}\left|S_{j} f(y)\right| d y
$$

independent of $f$-provided $j \geq k$. Hence

$$
\sup _{\ell \in \mathbb{Z}^{n}} \int_{Q_{k, \ell}}\left|S_{j} f(y)\right| d y \leq c \sup _{\ell \in \mathbb{Z}^{n}} \int_{Q_{k, \ell}} \frac{\left|S_{j} f(y)\right|^{2}}{S_{j}^{*} f(y)} d y .
$$

Combining this inequality and (6.6) we finally derive

$$
\left\|f\left|M\left(B_{\infty, \infty}^{0}\right) \| \geq C_{4} \sup _{k=0,1, \ldots} \sup _{\ell \in \mathbb{Z}^{n}} 2^{k n} \int_{Q_{k, \ell}} \sum_{j=k}^{\infty}\right| S_{j} f(y) \mid d y\right.
$$

for some constant $C_{4}$ independent of $f$. This proves the claim.

None of the three conditions characterizing $M\left(B_{\infty, \infty}^{0}\right)$ can be omitted.

Lemma 13 1. There exists $f \in L_{\infty}$ with $\sup _{j=1, \ldots} j\left\|S_{j} f \mid L_{\infty}\right\|<\infty$ but $f \notin F_{\infty, 1}^{0}$.

2. There exists $f \in L_{\infty} \cap F_{\infty, 1}^{0}$ with $\sup _{j=1, \ldots} j\left\|S_{j} f \mid L_{\infty}\right\|=\infty$.

3. There exists $f \in F_{\infty, 1}^{0}$ with $\sup _{j=1, \ldots} j\left\|S_{j} f \mid L_{\infty}\right\|<\infty$ but $f \notin L^{\infty}$.

Proof. Step 1. Claim 1 is a consequence of the apparently much stronger statement that there is a bounded function $f$ with $\sup _{j} j\left\|S_{j} f \mid L_{\infty}\right\|<\infty$, for which $\phi f$ is not in $B_{1,1}^{0}$ for a smooth cutoff function $\phi$.

It suffices to construct an example for $n=1$. If $n>1$ we use the function constructed below as function of one coordinate. We choose

$$
f(x)=\exp \left(i \sum_{j=1}^{\infty}(M j)^{-1} \cos \left(2^{M j} x\right)\right)
$$


where $M$ is a large integer to be chosen later. The function $f$ is bounded. Let

$$
g_{k}(x)=\exp \left(i \sum_{j=1}^{k}(M j)^{-1} \cos \left(2^{M j} x\right)\right) .
$$

As we shall see below the $g_{k}$ approximate $f$ in $\mathcal{S}^{\prime}$.

Substep 1.1 We have

$$
\left\|g_{k}^{\prime} \mid L_{\infty}\right\| \leq \frac{6}{M k} 2^{k M}, \quad M \geq 1,
$$

and hence

$$
\left\|S_{j} g_{k} \mid L_{\infty}\right\| \leq c \min \left(1,2^{M k-j} \frac{1}{M k}\right)
$$

Let $f_{1}=g_{1}$ and

$$
\begin{aligned}
f_{k}(x) & =g_{k}(x)-g_{k-1}(x)=g_{k-1}(x)\left(e^{\frac{i}{M k} \cos \left(2^{M k} x\right)}-1\right) \\
& =g_{k-1}(x)\left(\frac{i}{M k} \cos \left(2^{M k} x\right)+R_{k}(x)\right), \quad k \geq 2 .
\end{aligned}
$$

Clearly

Further

$$
\left\|R_{k} \mid L_{\infty}\right\| \leq \frac{e}{M^{2} k^{2}}
$$

$$
\begin{aligned}
S_{j} f_{k}(x) & =g_{k-1}(x) S_{j}\left(\frac{i}{M k} \cos \left(2^{M k} \cdot\right)\right)(x)+g_{k-1}(x) S_{j} R_{k}(x) \\
& +\left[S_{j}, g_{k-1}\right]\left(\frac{i}{M k} \cos \left(2^{M k} y\right)+R_{k}(y)\right)(x)
\end{aligned}
$$

The commutator $\left[S_{j}, g_{k-1}\right]$ can be estimated as follows

$$
\begin{aligned}
& \left|\left[S_{j}, g_{k-1}\right]\left(\frac{i}{M k} \cos \left(2^{M k} y\right)+R_{k}(y)\right)(x)\right| \\
& \leq \int\left|\psi_{1}(z)\right|\left|g_{k-1}\left(x-2^{-j} z\right)-g_{k-1}(x)\right| \\
& \quad \times\left|\frac{i}{M k} \cos \left(2^{M k}\left(x-2^{-j} z\right)\right)+R_{k}\left(x-2^{-j} z\right)\right| d z \\
& \leq c 2^{M(k-1)-j}(M k)^{-2}
\end{aligned}
$$

where we used estimate (6.7). Together with $S_{M k}\left(\cos \left(2^{k M} \cdot\right)(x)=\cos \left(2^{M k} x\right)\right.$ this implies

$$
\left\|S_{M k} f_{k}\left|L_{1}(B(0,1)) \| \geq \int_{|x| \leq 1}\right| \frac{i}{M k} \cos \left(2^{M k} x\right) \mid d x-\frac{c}{(M k)^{2}} \geq \frac{C}{M k} .\right.
$$


Substep 1.2 Obviously,

$$
\left\|f_{k}^{\prime} \mid L_{\infty}\right\| \leq c \frac{2^{k M}}{k M}
$$

(cf. (6.7)) and hence

$$
\left\|S_{j} f_{k} \mid L_{\infty}\right\| \leq c \min \left\{1, \frac{2^{M k-j}}{M k}\right\} .
$$

The aim of this substep is to improve this estimate for $j$ small. Let

$$
g_{k-1}=S^{M(k-1)} g_{k-1}+\left(g_{k-1}-S^{M(k-1)} g_{k-1}\right) .
$$

Furthermore, by (6.8)

$$
\begin{aligned}
\left\|g_{k-1}-S^{M(k-1)} g_{k-1} \mid L_{\infty}\right\| & \leq \sum_{j=M k-M+1}^{\infty}\left\|S_{j} g_{k-1} \mid L_{\infty}\right\| \\
& \leq c \sum_{j=M k-M+1}^{\infty} 2^{M(k-1)-j} \frac{1}{M(k-1)} \\
& \leq \frac{c}{M k} .
\end{aligned}
$$

Then, by checking the supports of the Fourier transforms

$$
S_{j}\left[\left(S^{M(k-1)} g_{k-1}\right)(x) \frac{i}{M k} \cos \left(2^{M k} x\right)\right]=0
$$

if $j \leq M k-3$. By our previous estimate this leads to (still assuming $j \leq M k-3)$

$$
\begin{aligned}
& \left\|S_{j}\left(g_{k-1}(y) \frac{i}{M k} \cos \left(2^{M k} y\right)\right) \mid L_{\infty}\right\| \\
& \leq\left\|S_{j}\left(g_{k-1}(y) \frac{i}{M k} \cos \left(2^{M k} y\right)-\left(S^{M(k-1)} g_{k-1}\right)(y) \frac{i}{M k} \cos \left(2^{M k} y\right)\right) \mid L_{\infty}\right\| \\
& \quad+\left\|S_{j}\left(\left(S^{M(k-1)} g_{k-1}\right)(y) \frac{i}{M k} \cos \left(2^{M k} y\right)\right) \mid L_{\infty}\right\| \\
& \leq c\left\|\left(g_{k-1}(y)-S^{M(k-1)} g_{k-1}(y)\right) \frac{i}{M k} \cos \left(2^{M k} y\right) \mid L_{\infty}\right\| \\
& \leq \frac{C}{M^{2} k^{2}} .
\end{aligned}
$$

Altogether, if $j \leq M k-3$,

$$
\left\|S_{j} f_{k}\left|L_{\infty}\left\|\leq \frac{c}{M^{2} k^{2}}+\right\| S_{j}\left(g_{k-1} R_{k}\right)\right| L_{\infty}\right\| \leq \frac{C}{M^{2} k^{2}} .
$$


Substep 1.3 Our estimates (6.13), (6.14) and (6.10) are sufficient for convergence of $g_{k}$ in $B_{\infty, q}^{0}$ for all $q>1$ : The functions $g_{k}$ are uniformly bounded in $B_{\infty, q}^{0} \cap L_{\infty}$. Moreover

$$
\left\|S_{j}\left(g_{k+N}-g_{k}\right)\left|L_{\infty}\|=\| \sum_{\ell=k+1}^{N} S_{j} f_{\ell}\right| L_{\infty}\right\| \leq \frac{C}{M k},
$$

where $C$ does not depend on $N, k$ and $M$ if $M k-3>j$. This justifies

$$
S_{M k} f=S_{M k} g_{k-1}+S_{M k} f_{k}+S_{M k}\left(\sum_{\ell=k+1}^{\infty} f_{\ell}\right)
$$

in $L_{\infty}$. By $(6.14)$

$$
\left\|S_{M k}\left(\sum_{\ell=k+1}^{\infty} f_{\ell}\right) \mid L_{\infty}\right\| \leq \frac{c}{M^{2} k}
$$

and

$$
\left\|S_{M k} g_{k-1} \mid L_{\infty}\right\| \leq \frac{c}{M k} 2^{-M}
$$

which follows by writing $g_{k-1}=\sum_{j=1}^{k-1} f_{j}$ and using (6.13). Altogether we arrive at

$$
\left\|S_{M k} f \mid L_{1}(B(0,1))\right\| \geq \frac{c}{M k}
$$

for $M$ sufficiently large using (6.11), the two previous estimates and

$$
\left\|S_{j} f \mid L_{\infty}\right\| \leq \frac{c}{j}
$$

by making use of (6.13) and (6.14). This implies the first assertion.

Step 2. Let $\varrho \in \mathcal{S}$ be a function such that $\varrho(0)=1$ and $\operatorname{supp} \mathcal{F} \varrho \subset$ $\left\{\xi: \frac{3}{2} \leq|\xi| \leq 2\right\}$. Then we have

$$
S_{j} \varrho\left(2^{k-1} \cdot\right)(x)=\delta_{j, k} \varrho\left(2^{k-1} x\right), \quad j=0,1, \ldots, \quad k=1,2, \ldots .
$$

We define

$$
f(x)=\sum_{k=1}^{\infty} k^{-2} \varrho\left(2^{2^{k}} x\right)
$$

it follows $f \in B_{\infty, 1}^{0} \hookrightarrow L_{\infty} \cap F_{\infty, 1}^{0}$ but

$$
\sup _{k \in \mathbb{N}} k\left\|S_{k} f(x)\left|L_{\infty}\left\|=\sup _{k \in \mathbb{N}}\left(2^{k}+1\right)\right\| k^{-2} \varrho\left(2^{2^{k}} x\right)\right| L_{\infty}\right\|=\infty .
$$


Step 3. It remains to construct $f$ satisfying Claim 3.

Step 3.1 To prepare our argument we start with the following claim first: given $\varepsilon>0$ there exists $h_{\varepsilon} \in C^{\infty}$ with

$$
\sup _{j=0,1, \ldots}(1+j)\left\|S_{j} h_{\varepsilon}\left|L_{\infty}\|\leq \varepsilon, \quad\| h_{\varepsilon}\right| F_{\infty, 1}^{0}\right\| \leq \varepsilon
$$

and

$$
\sup _{x \in \mathbb{R}^{n}}\left|h_{\varepsilon}(x)\right|=h_{\varepsilon}(0) \geq 1
$$

We put, using $\varrho$ from the previous step

$$
h_{M, N}(x)=\sum_{k=M}^{N} k^{-1}(\ln k)^{-1} \varrho\left(2^{k-1} x\right), \quad 2 \leq M<N .
$$

We have

$$
\left\|h_{M, N}\left|B_{2, \infty}^{n / 2}\left\|=2^{n / 2} M^{-1}(\ln M)^{-1}\right\| \varrho\right| L_{2}\right\|,
$$

cf. [21, 4.6.2]. Marschall [14] showed $B_{2, \infty}^{n / 2} \hookrightarrow F_{\infty, 1}^{0}$. We observe that if the Fourier transform of $\varrho$ is nonnegative it assumes its maximum at $x=0$, hence

$$
\sup (1+j)\left\|S_{j} h_{M, N} \mid L_{\infty}\right\|=\frac{(1+M)}{M \ln M},
$$

cf. (6.15). Finally starting with $M$ such that (6.17) is satisfied ( $M \sim e^{1 / \varepsilon}$ ) we may choose $N$ in dependence on $M$ such that also (6.18) is fulfilled. Step 3.2 We define

$$
f_{M}=\sum_{j=0}^{M} h_{2^{-j}}
$$

Then $f_{M}(0) \geq M+1$ and

$$
\sup _{j=0,1 \ldots}(1+j)\left\|S_{j} f_{M} \mid L_{\infty}\right\| \leq 2 .
$$

Now we claim that, if

$$
\sup _{j=0,1 \ldots}(1+j)\left\|S_{j} f \mid L_{\infty}\right\|<\infty
$$

implies $f \in L_{\infty}$ then there exists a constant $C>0$ with

$$
\left\|f\left|L_{\infty}\left\|\leq C \sup _{j=0,1 \ldots}(1+j)\right\| S_{j} f\right| L_{\infty}\right\| .
$$


The existence of $f_{M}$ implies that such a constant cannot exist and thus Claim 3 is true. We prove the claim. Let $\mathbf{B}$ be the Banach space of tempered distributions for which the norm on the right hand side is finite. We suppose that $f \in \mathbf{B}$ implies $f \in L_{\infty}$. Now we consider the sequence of operators

$$
T_{j}: \mathbf{B} \ni f \rightarrow S^{j} f \in L_{\infty}, \quad j=0,1, \ldots
$$

Clearly

$$
\sup _{j}\left\|T_{j} f\left|L_{\infty}\|\leq c\| f\right| L_{\infty}\right\| \leq c_{f}\|f \mid \mathbf{B}\| .
$$

From the pointwise boundedness we derive uniform boundedness of the sequence $T_{j}$ and thus, by the Fatou property of $L_{\infty}$

$$
\left\|f\left|L_{\infty}\left\|\leq \sup _{j}\right\| S^{j} f\right| L_{\infty}\right\| \leq \sup _{j}\left\|T_{j}\right\|\|f \mid \mathbf{B}\|
$$

for all $f \in \mathbf{B}$.

\subsection{The characterization of $M\left(B_{\infty, 1}^{0}\right)$ - Proof of Theorem 5}

As in the preceeding section we first give a proof of the main theorem. We verify afterwards that the conditions in the characterization of the multipliers are independent.

Proof of Theorem 5. Thanks to Lemma 10(i) we have $M\left(B_{\infty, 1}^{0}\right)=$ $M\left(B_{1, \infty}^{0}\right)$. So we may deal with $M\left(B_{1, \infty}^{0}\right)$ instead of $M\left(B_{\infty, 1}^{0}\right)$.

Step 1. The estimate of $\Pi_{1}$ follows from $B_{\infty, 1}^{0} \hookrightarrow L_{\infty}$ and (5.4).

Step 2. We have

$$
\begin{aligned}
\left\|\Pi_{2}(f, g) \mid B_{1, \infty}^{0}\right\| & \leq c \sup _{j=0,1, \ldots}\left\|S_{j}\left(\sum_{k=0}^{\infty} \sum_{\ell=k-1}^{k+1} S_{\ell} f S_{k} g\right) \mid L_{1}\right\| \\
& \leq c \sup _{j=0,1, \ldots} \sum_{k=j-3}^{\infty} \sum_{\ell=k-1}^{k+1}\left\|S_{\ell} f S_{k} g \mid L_{1}\right\| \\
& \leq c\left\|f\left|B_{\infty, 1}^{0}\|\| g\right| B_{1, \infty}^{0}\right\| .
\end{aligned}
$$

with $c$ independent of $f$ and $g$.

Step 3. Let $Q_{\ell, k}$ be the dyadic cubes defined in (2.1). We recall that the maximal function $S_{j}^{*}$ has been defined in the proof of Theorem 4. Arguing as above with the support of the Fourier transform we see that

$$
\left\|\Pi_{3}(f, g)\left|B_{1, \infty}^{0}\left\|\leq c \sup _{j=0,1,2, \ldots}\right\| S_{j} f S^{j-2} g\right| L_{1}\right\| .
$$


Using the maximal inequality stated in Proposition 23 we find

$$
\begin{aligned}
\int_{Q_{0,0}} \mid S_{j} f & S^{j-2} g\left|d x \leq c \sum_{\ell=0}^{j-2} \sum_{Q_{l, t} \subset Q_{0,0}} \sup _{x \in Q_{\ell, t}}\right| S_{\ell} g(x)\left|\int_{Q_{\ell, r}}\right| S_{j} f(y) \mid d y \\
& \leq c \sum_{l=0}^{j-2} \sum_{Q_{l, r} \subset Q_{0,0}} 2^{\ell n} \int_{Q_{\ell, t}}\left|S_{\ell}^{*} g(x)\right| d x \int_{Q_{\ell, t}}\left|S_{j} f(y)\right| d y \\
& \leq c \sum_{\ell=0}^{j-2} 2^{\ell n} \max _{Q_{\ell, r} \subset Q_{0,0}} \int_{Q_{\ell, r}}\left|S_{j} f(y)\right| d y \int_{Q_{0,0}}\left|S_{\ell}^{*} g(x)\right| d x
\end{aligned}
$$

The same argument applies for all cubes $Q_{0, t}, t \in \mathbb{Z}^{n}$. We take the supremum with respect to $j$ and sum over all cubes $Q_{0, t}$ to arrive at

$$
\left\|\Pi_{3}(f, g)\left|B_{1, \infty}^{0}\left\|\leq c \sup _{j=\mathbb{N}, t \in \mathbb{Z}^{n}} \sum_{\ell=0}^{j-2} 2^{\ell n} \max _{Q_{\ell, r} \subset Q_{0, t}} \int_{Q_{\ell, r}}\left|S_{j} f(y)\right| d y\right\| g\right| B_{1, \infty}^{0}\right\|
$$

with $c$ independent of $f$ and $g$. The estimate

$$
\left\|f \mid M\left(B_{\infty, 1}^{0}\right)\right\| \leq c\left(\left\|f\left|B_{\infty, 1}^{0} \|+\sup _{j \geq 2, t \in \mathbb{Z}^{n}} \sum_{l=0}^{j-2} 2^{l n} \max _{Q_{l, r} \subset Q_{0, t}} \int_{Q_{l, r}}\right| S_{j} f(y) \mid d y\right)\right.
$$

follows from (5.4), (6.19), and (6.20).

Step 4. Necessity. Because of $1 \in B_{\infty, 1}^{0}$, cf. Lemma 22, $f \in B_{\infty, 1}^{0}$ is a necessary condition.

To prove the other part we begin with the construction of useful functions. For a dyadic cube $Q_{\ell, r}$ the function $\chi_{\ell, r}$ denotes its characteristic function.

Proposition 14 There exists $c_{0}>0$ such that for each natural number $j$ and each sequence of dyadic cubes $\left\{Q_{\ell, r_{\ell}}\right\}_{\ell=0}^{j}$ there exists $g \in B_{1, \infty}^{0}$ satisfying

$$
\begin{gathered}
\left\|g\left|B_{1, \infty}^{0}\left\|\leq c_{0}, \quad \sum_{k=j-1}^{\infty}\right\| S_{k} g\right| L_{1}\right\| \leq c_{0} \\
\frac{1}{6} \sum_{\ell} 2^{\ell n} \chi_{\ell, r_{\ell}}(x) \leq|g(x)| \leq \sum_{\ell} 2^{\ell n} \chi_{\ell, r_{\ell}}(x) .
\end{gathered}
$$

Proof. Step 1. Preparations. Let $Q$ be the unit cube centered at the origin and with sides parallel to the axes. Let $P=\left\{x:\left|x_{i}\right| \leq a, i=1, \ldots, n\right\}$ with $(2 a)^{n}=1 / 2$. Further, let

$$
h(x)=\chi_{Q}(x)-2 \chi_{P}(x) .
$$


Then $|h(x)| \leq 1$ for all $x \in \mathbb{R}^{n}$. We claim

$$
\left\|2^{\ell n} S_{j}\left(h\left(2^{\ell} y\right)\right)(x) \mid L_{1}\right\| \leq c_{2} 2^{-|j-\ell|}
$$

for some $c_{2}$ independent of $j$ and $\ell$. After scaling we may assume that $\ell=0$ and that $j$ is an integer. Suppose that $j \leq 0$. Then

$$
\left|2^{j n} \iint \psi_{1}\left(2^{j}(x-y)\right) h(y) d y d x\right| \leq c 2^{j} \int|x| \sup _{y \in B\left(x, 2^{j}\right)}\left|\nabla \psi_{1}(y)\right| d x \leq c 2^{j}
$$

because the mean of $h$ vanishes. The case $j>0$ is simpler. Here the convolution is essentially supported in a $2^{-j}$ neighborhood of the jumps of $h$. It is uniformly bounded, hence the estimate in that case.

Step 2. Let $z_{j, r}$ denote the center of $Q_{j, r}$. We define

$$
g(x)=\sum_{\ell=1}^{j} i^{\ell} 2^{\ell n} h\left(2^{\ell}\left(x-z_{\ell, r_{\ell}}\right)\right)
$$

Then

$$
\left\|g \mid B_{1, \infty}^{0}\right\| \leq \sup _{t=0, \ldots} \sum_{\ell=1}^{j} c_{2} 2^{-|\ell-t|} \leq 3 c_{2},
$$

independent of $j$ and the chosen sequence $\left\{r_{\ell}\right\}_{\ell=1}^{j}$. Similarly, for $k \geq j$,

$$
\left\|S_{k} g \mid L_{1}\right\| \leq \sum_{\ell=1}^{j} c_{2} 2^{\ell-k} \leq 2 c_{2} 2^{j-k} .
$$

Taking $c_{0}=7 c_{2}$ the first assertion of our proposition is proved. Moreover

$$
\left|\sum_{\ell=1}^{j} i^{\ell} 2^{\ell n} h\left(2^{\ell}\left(x-z_{\ell, r_{\ell}}\right)\right)\right| \leq \sum_{\ell=1}^{j} 2^{\ell n} \chi_{\ell, r_{\ell}}(x) .
$$

Let $M \leq j$ be even and let $x \in Q_{M, r_{M}}$. Then, with $z_{l}=z_{\ell, r_{\ell}}$ and taking into account the phase,

$$
\left|\sum_{\ell=1}^{M} i^{\ell} 2^{\ell n} h\left(2^{\ell}\left(x-z_{\ell}\right)\right)\right| \geq 2^{n M}-2 \sum_{\ell=1}^{M / 2-1} 2^{2 n \ell} \geq \frac{1}{3} 2^{n M} \geq \frac{1}{6} \sum_{\ell=1}^{M} 2^{\ell n} .
$$

The case $M$ odd is proven in the same way. Hence the claim.

In what follows we suppose that $g$ is as in Proposition 14. As above we see

$$
\left\|f\left|M\left(B_{1, \infty}^{0}\right)\|\| g\right| B_{1, \infty}^{0}\right\| \geq c_{3} \sup _{j=2,3, \ldots}\left\|S_{j} f S^{j-2} g \mid B_{1, \infty}^{0}\right\|
$$


Next we want to switch from the norm in $B_{1, \infty}^{0}$ to the norm in $L_{1}$. We choose $g$ in dependence of $j$ as in Proposition 14. In this particular situation we have

$$
\begin{aligned}
\left\|S_{j} f g \mid L_{1}\right\| & \leq\left\|S_{j} f\left|L_{\infty}\left\|\left(\sum_{\ell=j-1}^{\infty}\left\|S_{\ell} g \mid L_{1}\right\|\right)+\right\| S_{j} f S^{j-2} g\right| L_{1}\right\| \\
& \leq c_{4}\left\|S_{j} f\left|L_{\infty}\|+\| S_{j} f S^{j-2} g\right| B_{1, \infty}^{0}\right\| \\
& \leq c_{5}\left\|S_{j} f\left|M\left(B_{\infty, 1}^{0}\right)\|\| g\right| B_{1, \infty}^{0}\right\|, \\
& \leq c_{6}\left\|f \mid M\left(B_{\infty, 1}^{0}\right)\right\| .
\end{aligned}
$$

Here the second inequality holds because of supp $\mathcal{F}\left(S_{j} f S^{j-2} g\right)$ is contained in $\left\{\xi: 2^{j-2} \leq|\xi| \leq 2^{j+2}\right\}$. Hence

$$
\frac{1}{6} \sum_{\ell=1}^{j-1} 2^{\ell n} \int_{Q_{\ell, r_{\ell}}}\left|S_{j} f(x)\right| d x \leq\left\|S_{j} f g\left|L_{1}\left\|\leq c_{6}\right\| f\right| M\left(B_{1, \infty}^{0}\right)\right\|,
$$

which completes the proof of necessity of the conditions.

Remark 15 Obviously, if $f \in B_{\infty, 1}^{0}$ and if $\sup _{j=1,2, \ldots} j\left\|S_{j} f \mid L_{\infty}\right\|<\infty$, then $f \in M\left(B_{1, \infty}^{0}\right)$ follows.

As before the conditions characterizing $M\left(B_{\infty, 1}^{0}\right)$ are independent of each other.

Lemma 16 (i) There exists a function $f \in B_{\infty, 1}^{0}$ such that

$$
\sup _{j \in \mathbb{N}} \sup _{x \in \mathbb{R}^{n}} \sum_{k=1}^{j} \sup _{|y-x| \leq 1} 2^{k n} \int_{B\left(y, 2^{-k}\right)}\left|S_{j} f(z)\right| d z=\infty .
$$

(ii) There exists a function $f \in L_{\infty}$ such that $f \notin B_{\infty, 1}^{0}$ but

$$
\sup _{j \in \mathbb{N}} \sup _{x \in \mathbb{R}^{n}} \sum_{k=1}^{j} \sup _{|y-x| \leq 1} 2^{k n} \int_{B\left(y, 2^{-k}\right)}\left|S_{j} f(z)\right| d z<\infty .
$$

Proof. Step 1. Let

$$
f(x)=\sum_{k=2}^{\infty}(\ln k) k^{-2} e^{i 2^{k^{2}} x}
$$


This function belongs to $B_{\infty, 1}^{0}$ but

$$
\sum_{\ell=0}^{k^{2}} 2^{\ell n} \int_{Q_{\ell, 0}}\left|S_{k^{2}} f(z)\right| d z=\ln k
$$

Step 2. To prove part (ii) we consider $f(x)=\sum_{j=1}^{\infty} \varrho\left(2^{j-1} x-x^{j}\right)$, where $\varrho$ is as in the proof of Lemma 13. Choosing $x^{j}$ appropriate $f$ belongs to $L_{\infty}$. Obviously, $f$ does not belong to $B_{\infty, 1}^{0}$. Furthermore

$$
\int_{Q_{\ell, r}}\left|S_{j} f(y)\right| d y \leq 2^{-(j-1) n}\left\|\varrho \mid L_{1}\right\|
$$

and this guarantees (6.22).

\section{The relation to continuous functions}

\subsection{Discontinuous functions in $M\left(B_{\infty, \infty}^{0}\right)$ and $M(b m o)$}

It is of certain interest to clarify whether multipliers in $M\left(B_{\infty, \infty}^{0}\right)$ or $M(b m o)$ are necessarily continuous. This is clearly true for $M\left(B_{\infty, 1}^{0}\right)$.

Proposition 17 We have

$$
f(x)=\cos (\ln (1+|\ln (|x|)|)) \in M(b m o) \cap M\left(B_{\infty, \infty}^{0}\right) .
$$

Proof. We shall see below that $M(b m o) \hookrightarrow M\left(B_{\infty, \infty}^{0}\right)$. Hence it suffices to show that $f \in M(b m o)$. We calculate

$$
\left|\frac{\partial f}{\partial x_{j}}(x)\right| \leq\left|\frac{x_{j}}{|x|^{2}(1+|\ln (|x|)|)}\right|, \quad j=1,2, \ldots, n .
$$

Suppose $|x| \geq 2 R$. Then, by Poincaré's inequality

$$
\int_{B_{R}(x)}\left|f(y)-f_{B_{R}(x)}\right| d y \leq c R \int_{B_{R}(x)}|\nabla f(y)| d y \leq c R^{n}(1+|\ln R|)^{-1}
$$


Now suppose that $x=0$ and $R \leq 1$. If $n \geq 2$ then the argument works without change. If $n=1$ we use a Hardy type estimate instead:

$$
\begin{aligned}
(2 R)^{-1} \int_{-R}^{R} \mid f-(2 R)^{-1} & \int_{-R}^{R} f d y\left|d x=R^{-1} \int_{0}^{R}\right| f-R^{-1} \int_{0}^{R} f d y \mid d x \\
& \leq R^{-2} \int_{0}^{R} \int_{0}^{R}|f(x)-f(y)| d x d y \\
& \leq 2 R^{-2} \int_{0}^{R} \int_{x}^{R}|f(x)-f(y)| d x d y \\
& \leq 2 R^{-2} \int_{0}^{R} \int_{x}^{R} \int_{x}^{y}\left|f^{\prime}(z)\right| d z d y d x \\
& \leq 2 R^{-2} \int_{0}^{R} z(R-z)\left|f^{\prime}(z)\right| d z \\
& \leq 2 c R^{-2}(1+|\ln R|)^{-1} \int_{0}^{R}(R-z) d z \\
& \leq c(1+|\ln R|)^{-1} .
\end{aligned}
$$

It is not hard to verify the desired estimates for all the remaining balls: if $|x| \leq 2 R \leq 1$, we compute with $\tilde{B}=B(0,3 R)$, using the estimate for $x=0$,

$$
\begin{aligned}
\int_{B(x, R)}\left|f(y)-f_{B(x, R)}\right| d y & \leq c \int_{B(x, R)}\left|f(y)-f_{\tilde{B}}\right| d y \\
& \leq c \int_{\tilde{B}}\left|f(y)-f_{\tilde{B}}\right| d y \\
& \leq c R^{-n}(1+|\ln R|)^{-1}
\end{aligned}
$$

This implies $f \in M(b m o)$ by the characterization (1.6).

Next we will show that the discontinuity of elements of $M\left(B_{\infty, \infty}^{0}\right)$ must be weak in a certain sense. To this end we consider extremely simple discontinuous functions. Let $A$ be a measurable set and let $\chi_{A}$ be its characteristic function.

Proposition 18 Let $A$ be a nontrivial measurable set and denote by $\chi_{A}$ its characteristic function.

(i) $\chi_{A}$ does not belong to $M\left(B_{\infty, \infty}^{0}\right)$.

(ii) $\chi_{A}$ does not belong to $M(b m o)$.

To prepare the proof we add the following observation. 
Lemma 19 Let $f \in L_{\infty}$ be such that

$$
\sup _{j=0,1, \ldots}(1+j)\left\|S_{j} f \mid L_{\infty}\right\|<\infty .
$$

Then, for all $j \geq 1$,

$$
\sup _{x \in \mathbb{R}^{n}} \sup _{j=0,1, \ldots} j^{1 / 2} 2^{j n} \int_{B\left(x, 2^{-j}\right)}\left|f(y)-f_{B\left(x, 2^{-j}\right)}\right| d y<\infty .
$$

Proof. Step 1. First we recall that $S_{j}=\left(S_{j-1}+S_{j}+S_{j+1}\right) S_{j}$ and $\nabla\left(S_{j} f\right)=\left(\nabla \psi_{j}\right) * f$ hence

$$
\sup _{|y-z|<2^{-j}}\left|S_{k} f(y)-S_{k} f(z)\right| \leq C 2^{k-j}\left\|S_{k} f \mid L_{\infty}\right\|
$$

and

$$
2^{j n} \int_{B\left(x, 2^{-j}\right)}\left|S_{k} f(y)-\left(S_{k} f\right)_{B\left(x, 2^{-j}\right)}\right| d y \leq C 2^{k-j}\left\|S_{k} f \mid L_{\infty}\right\|,
$$

from which we derive that

$$
2^{j n} \int_{B\left(x, 2^{-j}\right)}\left|S^{j} f(y)-\left(S^{j} f\right)_{B\left(x, 2^{-j}\right)}\right| d y \leq c_{1} \sum_{k=0}^{j} 2^{k-j} \frac{1}{k+1} \leq \frac{c_{2}}{j}
$$

using our assumption on $f$. Here $c_{2}$ does not depend on $j$.

Step 2. We have, for $j \geq 2$,

$$
\left\|\sum_{k=j}^{\infty} S_{k} f \mid b m o\right\| \sim\left(\sup _{x, \ell} 2^{\ell n} \int_{B\left(x, 2^{-\ell}\right)} \sum_{k \geq \max (j, \ell)}\left|S_{k} f\right|^{2} d x\right)^{1 / 2}
$$

since the two norms are equivalent (recall that $S_{0} S_{k} f=0$ for $k \geq 2$ ). Hence

$$
\begin{aligned}
\left\|f-S^{j} f \mid b m o\right\|^{2} & \leq c \sup _{x, \ell \geq j} 2^{\ell n} \int_{B\left(x, 2^{-\ell}\right)} \sum_{k \geq \max (j, \ell)}\left|S_{k} f\right|^{2} d x \\
& \leq \bar{c} \sum_{k=j}^{\infty}(1+k)^{-2} \sup _{\ell}(1+\ell)^{2}\left\|S_{\ell} f \mid L_{\infty}\right\|^{2} \\
& \leq \bar{c} j^{-1} \sup _{\ell}(1+\ell)^{2}\left\|S_{\ell} f \mid L_{\infty}\right\|^{2}
\end{aligned}
$$

which implies the desired estimate. 
Proof of Proposition 18. Since $\chi_{A}$ is locally integrable almost all points of $\mathbb{R}^{n}$ are Lebesgue points of $\chi_{A}$. Hence

$$
\lim _{\varepsilon \rightarrow 0} c_{n}^{-1} \varepsilon^{-n} \int_{B(x, \varepsilon)} \chi_{A}(y) d y=\left\{\begin{array}{l}
1 \\
0
\end{array} \quad \text { almost everywhere } .\right.
$$

For each $\varepsilon>0$ we may select two Lebesgue points $x_{1}(\varepsilon), x_{2}(\varepsilon)$ of $\chi_{A}$ such that

$$
c_{n}^{-1} \varepsilon^{-n} \int_{B\left(x_{1}(\varepsilon), \varepsilon\right)} \chi_{A}(y) d y \geq \frac{2}{3} \quad \text { and } \quad c_{n}^{-1} \varepsilon^{-n} \int_{B\left(x_{2}(\varepsilon), \varepsilon\right)} \chi_{A}(y) d y \leq \frac{1}{3} .
$$

The function $g(x)=\int_{B(x, \varepsilon)} \chi_{A}(y) d y$ is continuous. Consequently, on the way from $x_{1}(\varepsilon)$ to $x_{2}(\varepsilon)$ we find a point $x_{\varepsilon}$ such that

$$
c_{n}^{-1} \varepsilon^{-n} \int_{B\left(x_{\varepsilon}, \varepsilon\right)} \chi_{A}(y) d y=\frac{1}{2} .
$$

But this implies

$$
c_{n}^{-1} \varepsilon^{-n} \int_{B\left(x_{\varepsilon}, \varepsilon\right)}\left|\chi_{A}(y)-\left(\chi_{A}\right)_{B\left(x_{\varepsilon}, \varepsilon\right)}\right| d y=\frac{1}{2} .
$$

The equation (7.3) immediately implies (i). The second assertion is a consequence of the characterization of $M(b m o)$ since $M(b m o) \hookrightarrow M\left(B_{\infty, \infty}^{0}\right)$ by Lemma 21 below.

\subsection{Continuous functions in $M\left(B_{\infty, \infty}^{0}\right)$}

In what follows we are interested in large spaces of continuous functions which imbed into multiplier spaces. For the definition of the classes $C_{q}^{\varrho}$ see the Appendix.

Lemma 20 (i) We have $C_{q} \hookrightarrow M\left(B_{\infty, \infty}^{0}\right)$ if and only if $q=1$.

(ii) We have $C^{|\ln | \hookrightarrow M(b m o)} \hookrightarrow M\left(B_{\infty, \infty}^{0}\right)$.

Proof. The implication $C_{1} \hookrightarrow M\left(B_{\infty, \infty}^{0}\right)$ follows from $C_{1}=C_{D}, C_{D} \hookrightarrow B_{\infty, 1}^{0}$ of Lemma 30 and the trivial imbedding $B_{\infty, 1}^{0} \hookrightarrow F_{\infty, 1}^{0}$.

For the other direction we observe that $C_{q} \hookrightarrow C^{|\ln |}$ if and only if $q=1$ (Lemma 29) and the same arguments as there show that $C_{q} \hookrightarrow M\left(B_{\infty, \infty}^{0}\right)$ only if $q=1$.

The first assertion in (ii) follows directly from the definition. The second imbedding is part of Lemma 21. 


\section{The relation between $M(b m o), M\left(B_{\infty, \infty}^{0}\right)$ and $M\left(B_{\infty, 1}^{0}\right)$}

Lemma 21 (i) We have $M($ bmo $) \hookrightarrow M\left(B_{\infty, \infty}^{0}\right)$.

(ii) There exists a function in $\cap_{p, q} M\left(B_{p, q}^{0}\right)$ but not in $M(b m o)$.

(iii) There exists a function in $M($ bmo $)$ but not in $M\left(B_{\infty, 1}^{0}\right)$.

(iv) There exists a function in $M\left(B_{\infty, 1}^{0}\right)$ but not in $M\left(B_{\infty, \infty}^{0}\right)$.

Proof. Step 1. We recall that

$$
\left\|f|M(b m o)\|\sim\| f| L_{\infty}\right\|+\sup _{x} \sup _{R<1 / 2}|\ln R|\left(R^{-n} \int_{B(x, R)}\left|f-f_{B}\right|^{2} d x\right)^{1 / 2} .
$$

In Section 2 we may choose the dyadic partition of unity so that the inverse Fourier transforms $\psi_{j}$ have compact support - thereby loosing the compact support in the Fourier space and replacing it by fast decay. Then $S_{j}$ is the convolution with the function $\psi_{j}$ which is supported on a ball of size $c 2^{-j}$ with mean zero (if $j \geq 1$ ) and bounded $L_{1}$-norm. In addition we require $\int \psi_{0}(x) d x=1$. Hence for $j \geq 1$ by using $\int \psi_{j}(y) d y=0$ we find

$$
\left|S_{j} f(x)\right| \leq c 2^{j n / 2} \| f-f_{B} \mid L_{2}\left(B\left(x, c 2^{-j}\right)\left\|\leq c j^{-1}\right\| f \mid M(b m o) \| .\right.
$$

This shows that $f$ satisfies the third condition in (1.7).

Substep 2.1 We claim that

$$
\left\|f-S^{j} f\left|b m o\left\|\leq c j^{-1}\right\| f\right| M(b m o)\right\| .
$$

Clearly

$$
\left\|\nabla S^{j} f\left|L_{\infty}\left\|\leq c 2^{j} j^{-1}\right\| f\right| M(b m o)\right\|
$$

which implies the desired bound for all $k \geq j$ :

$$
2^{k n} \int_{B\left(x, 2^{-k}\right)}\left|S^{j} f(y)-S^{j} f_{B\left(x, 2^{-k}\right)}\right| d y \leq c j^{-1} 2^{j-k}\|f \mid M(b m o)\| .
$$

On the other hand

$$
2^{k n} \int_{B\left(x, 2^{-k}\right)}\left|f(y)-S^{j} f(y)\right| d y \leq c \sup _{x} 2^{j n} \int_{B\left(x, 2^{-j}\right)}\left|f(y)-S^{j} f(y)\right| d y .
$$

The function $\psi_{0}$ is radial and decreasing. We may write it as

$$
\psi_{0}(x)=\int_{0}^{\infty} h(r) \chi_{B(0, r)}(x) d r
$$


where $h(|x|)=-\partial_{r} \psi_{0}(x)$. In particular

$$
\int h d r=1
$$

and

$$
\begin{aligned}
I & :=\int_{B\left(x, 2^{-j}\right)}\left|f(z)-S^{j} f(z)\right| d z \\
& =2^{j n} \int_{B\left(x, 2^{-j}\right)}\left|\int_{\mathbb{R}^{n}} \psi_{0}\left(2^{j}(z-y)\right)(f(z)-f(y)) d y\right| d z \\
& =\int_{B\left(x, 2^{-j}\right)}\left|\int_{\mathbb{R}^{n}} \int_{0}^{\infty} h(r) \chi_{B(0, r)}(w)\left(f(z)-f\left(z-2^{-j} w\right)\right) d r d y\right| d z \\
& \leq 2^{j(n+1)} \int_{B\left(x, 2^{-j}\right)} \int_{0}^{\infty} h\left(2^{j} t\right) t^{n}\left|f(z)-f_{B(z, t)}\right| d t d z .
\end{aligned}
$$

The function $\psi_{0}$ has compact support. Hence the integration with respect to $t$ is restricted to $\left[0, c 2^{-j}\right]$. With $B=B\left(x, c 2^{-j}\right)$ this leads to

$$
\int_{B\left(x, 2^{-j}\right)}\left|f(z)-f_{B(z, t)}\right| d z \leq\left(1+t^{-n} 2^{-j n}\right) \int_{B}\left|f(z)-f_{B}\right| d z .
$$

Altogether we end up with

$$
\begin{aligned}
I & \leq \int_{B}\left|f(z)-f_{B}\right| d z \int_{0}^{\infty} h\left(2^{j} t\right) t^{n}\left(1+t^{-n} 2^{-j n}\right) 2^{j} d t \\
& \leq c 2^{-j n} \int_{B}\left|f(z)-f_{B}\right| d z .
\end{aligned}
$$

Consequently

$$
\begin{aligned}
2^{j n} \int_{B\left(x, 2^{-j}\right)}\left|f(z)-f_{B\left(x, 2^{-j}\right)}\right| d z & \leq c|B|^{-1} \int_{B}\left|f(z)-f_{B}\right| d z \\
& \leq c \frac{1}{j}\|f \mid M(b m o)\| .
\end{aligned}
$$

This proves our claim (8.1).

Substep 2.2 It remains to prove $M(b m o) \hookrightarrow F_{\infty, 1}^{0}$. Both spaces are characterized by a supremum over balls. Scaling shows that the worst case is the case when the radius of the ball is large (but smaller than $1 / 2$ lets say). Let $B$ be a ball of radius $1 / 2$ and $a_{j}=\int_{B}\left|S_{j} f\right| d x$. Then, by (8.1) we obtain

$$
\begin{aligned}
\left(\sum_{k=j}^{\infty} a_{k}^{2}\right)^{1 / 2} & \leq c\left(\sum_{k=j}^{\infty} \int_{B}\left|S_{k} f\right|^{2} d x\right)^{1 / 2} \\
& \leq c_{1}\left\|f-S^{j-1} f \mid b m o\right\| \\
& \leq c_{2} j^{-1}\|f \mid M(b m o)\| .
\end{aligned}
$$


Thus

$$
\begin{aligned}
\sum_{j=1}^{\infty} a_{j} & \leq 4 \sum_{j=1}^{\infty} j^{-\frac{3}{4}} \sum_{k=1}^{j} k^{-\frac{1}{4}} a_{j} \\
& \leq 4 \sum_{k=1}^{\infty} k^{-\frac{1}{4}} \sum_{j=k}^{\infty} j^{-\frac{3}{4}} a_{j} \\
& \leq 10 c_{1} \sum_{k=1}^{\infty} k^{-\frac{1}{4}} k^{-1} k^{-1 / 4}\|f \mid M(b m o)\| \\
& \leq 21 c_{1}\|f \mid M(b m o)\|
\end{aligned}
$$

where we used Hölder's inequality in the third step.

Step 3. Let

$$
f\left(x_{1}, \ldots, x_{n}\right)=\sum_{k=2}^{\infty} k^{-1}(\ln k)^{-2} \sin 2^{k} x_{1}
$$

Thanks to

$$
S_{k} f(x)=k^{-1}(\ln k)^{-2} \sin 2^{k} x_{1}, \quad k \geq 2,
$$

it becomes obvious that $f$ belongs to all spaces $M\left(B_{p, q}^{0}\right), 1 \leq p, q \leq \infty$, cf. (1.9). It remains to disprove $f \in M(b m o)$. We compute

$$
2^{j n} \int_{B\left(0,2^{-j}\right)} \sum_{k \geq j}\left|S_{k} f\right|^{2} d x \geq c \sum_{k=j}^{\infty} k^{-2}|\ln k|^{-4} \geq c j^{-\frac{3}{2}} .
$$

This implies by (8.1) that $f \notin M(b m o)$.

Step 3. We have seen that there exist discontinuous functions in $M(b m o)$ but not in $M\left(B_{\infty, 1}^{0}\right) \hookrightarrow B_{\infty, 1}^{0} \hookrightarrow C\left(\mathbb{R}^{n}\right)$.

Step 4. To prove part (i) one can use the function defined in (6.16).

\section{Appendix}

We collect a few properties of Besov and Triebel-Lizorkin spaces. If there is no appropriate reference we shall give proofs.

Lemma 22 The function $f \equiv 1$ belongs to $B_{p, q}^{0}$ if and only if $p=\infty$.

Proof The assertion follows from the identity

$$
\mathcal{F}^{-1}\left[\varphi_{j} \mathcal{F} 1\right](x)= \begin{cases}1 & \text { if } j=0 \\ 0 & \text { otherwise }\end{cases}
$$


For a function $\varrho \in \mathcal{S}$ satisfying $\varrho(y)=0$ if $|y|>d$ we put

$$
\varrho^{*, a} f(x)=\sup _{y \in \mathbb{R}^{n}} \frac{\mathcal{F}^{-1}[\varrho \mathcal{F}](x-y)}{1+|d y|^{a}}, \quad x \in \mathbb{R}^{n}, \quad f \in \mathcal{S}^{\prime} .
$$

This is a maximal function of Peetre-Fefferman-Stein type. Corresponding maximal inequalities are proved in several places. Here we need the following, cf. Triebel [30, 2.3.6].

Proposition 23 Let $1 \leq p \leq \infty$. Let $\left\{\varphi_{j}\right\}_{j=0}^{\infty}$ be the system defined in (2.3). Then there exists a constant $c$ such that

$$
\left\|\varphi_{j}^{*, a} f\left|L_{p}\|\leq c\| \mathcal{F}^{-1}\left[\varphi_{j} \mathcal{F} f\right]\right| L_{p}\right\|
$$

holds with $c$ independent of $f \in \mathcal{S}^{\prime}$ and $j$.

There is a large variety of generalizations even of spaces of Besov-LizorkinTriebel type, in particular in the Russian literature. Here we concentrate on classes in a certain sense close to spaces of smoothness zero. Recall

$$
\omega_{p}(t, f)=\sup _{|h|<t}\left\|f(x+h)-f(x) \mid L_{p}\right\|, \quad t>0 .
$$

Definition 24 Let $\varrho:(0,1] \rightarrow \mathbb{R}$ be a non-increasing positive function.

(i) Let $1 \leq p, q \leq \infty$. Then we put

$$
B_{p, q}^{\varrho}=\left\{f \in L_{p}:\left\|f\left|B_{p, q}^{\varrho}\|=\| f\right| L_{p}\right\|+\left(\int_{0}^{1}\left(\varrho(t) \omega_{p}(t, f)\right)^{q} \frac{d t}{t}\right)^{1 / q}<\infty\right\},
$$

if $q<\infty$ and

$$
B_{p, \infty}^{\varrho}=\left\{f \in L_{p}: \quad\left\|f\left|B_{p, \infty}^{\varrho}\|=\| f\right| L_{p}\right\|+\sup _{0<t<1} \varrho(t) \omega_{p}(t, f)<\infty\right\},
$$

if $q=\infty$.

(ii) In case $p=\infty$ we put $C_{q}^{\varrho}=B_{\infty, q}^{\varrho}$ and in particular, $C^{\varrho}=B_{\infty, \infty}^{\varrho}$.

(iii) If $\varrho \equiv 1$, then we put $C_{q}^{\varrho}=C_{q}$. If in addition $q=1$ we use $C_{1}=C_{D}$.

Remark 25 Spaces of type $B_{p, q}^{\varrho}$ have been investigated e.g. in Gol'dman $[8,9,10]$. A survey has been given by Lizorkin in a supplement to the russian translation of Triebel's book [31]. 
We shall prove a Fourier-analytical characterization of the classes $B_{p, q}^{\varrho}$ which seems to be of independent interest.

Proposition 26 Let $\varrho:(0, \infty) \rightarrow \mathbb{R}$ be a non-increasing positive function such that $\varrho(t)=1$ if $t \geq 1$. We suppose

$$
\sup _{1 \leq t<\infty} t^{-\alpha} \sup _{0<v<1} \frac{\varrho(v)}{\varrho(t v)}<\infty
$$

for some $0 \leq \alpha<1$. Then it holds

$$
B_{p, q}^{\varrho}=\left\{f \in L_{p}: \quad\left\|f \mid L_{p}\right\|+\left(\sum_{j=0}^{\infty} \varrho^{q}\left(2^{-j}\right)\left\|f-S^{j} f \mid L_{p}\right\|^{q}\right)^{1 / q}<\infty\right\}
$$

if $q<\infty$ in the sense of equivalent norms with the usual modifications if $q=\infty$.

Proof. Step 1. Following Nikol'skij (cf. e.g. [18, 5.2.1] or [24]) we have the existence of a function $g \in \mathcal{S}$ such that

$$
f(x)-S^{j} f(x)=\int_{0}^{\infty} g(r) r^{n-1} \int_{|\gamma|=1}\left(\Delta_{r \gamma 2^{-j}}^{1} f\right)(x) d \gamma d r
$$

holds for all $f \in L_{1}+L_{\infty}$ and all $j=0,1, \ldots$ Let $I_{0}=[0,1)$ and let $I_{\ell}=\left[2^{\ell-1}, 2^{\ell}\right), \ell=1,2, \ldots$ Then it follows from the generalized Minkowski inequality

$$
\begin{aligned}
\left\|f-S^{j} f \mid L_{p}\right\| & \leq\left\|\int_{0}^{\infty}|g(r)| r^{n-1} \int_{|\gamma|=1}\left|\Delta_{r \gamma 2^{-j}}^{1} f(x)\right| d \gamma d r \mid L_{p}\right\| \\
& \leq c_{N} \sum_{\ell=0}^{\infty} \int_{I_{\ell}} 2^{-\ell(N+1)} \omega_{p}\left(2^{\ell-j}, f\right) d r \\
& \leq c_{N} \sum_{\ell=0}^{\infty} 2^{-\ell N} \omega_{p}\left(2^{\ell-j}, f\right),
\end{aligned}
$$

where $N>1$ is at our disposal. For convenience, let us put

$$
M=\sup _{t \geq 1} t^{-\alpha} \sup _{0<v<1} \frac{\varrho(v)}{\varrho(t v)} .
$$


Using (9.3) it follows

$$
\begin{aligned}
\left(\sum_{j=0}^{\infty} \varrho^{q}\left(2^{-j}\right)\left\|f-S^{j} f \mid L_{p}\right\|^{q}\right)^{1 / q} & \\
\leq & c_{N} \sum_{\ell=0}^{\infty} 2^{-\ell N}\left(\sum_{j=0}^{\infty} \varrho^{q}\left(2^{-j}\right) \omega_{p}\left(2^{\ell-j}, f\right)^{q}\right)^{1 / q} \\
\leq & 2 c_{N} \sum_{\ell=0}^{\infty} 2^{-\ell N}\left(\sum_{j=0}^{\ell} \varrho^{q}\left(2^{-j}\right)\left\|f \mid L_{p}\right\|^{q}\right. \\
& \left.+\left(\sup _{k=\ell, \ldots} \frac{\varrho^{q}\left(2^{-k}\right)}{\varrho^{q}\left(2^{\ell-k}\right)}\right) \sum_{j=\ell+1}^{\infty} \varrho^{q}\left(2^{\ell-j}\right) \omega_{p}\left(2^{\ell-j}, f\right)^{q}\right)^{1 / q}
\end{aligned}
$$

Next we use

$$
\sum_{\ell=0}^{\infty} 2^{-\ell N}\left(\sum_{j=0}^{\ell} \varrho^{q}\left(2^{-j}\right)\right)^{1 / q} \leq M \sum_{\ell=0}^{\infty} 2^{-\ell N} 2^{\ell \alpha}\left(\frac{1}{1-2^{\alpha q}}\right)^{1 / q}
$$

and

$$
\begin{aligned}
& \left.\left(\sup _{k=\ell, \ldots} \frac{\varrho^{q}\left(2^{-k}\right)}{\varrho^{q}\left(2^{\ell-k}\right)}\right) \sum_{j=\ell+1}^{\infty} \varrho^{q}\left(2^{\ell-j}\right) \omega_{p}\left(2^{\ell-j}, f\right)^{q}\right)^{1 / q} \\
& \quad \leq M 2^{\ell \alpha+1}\left(\int_{0}^{1} \varrho^{q}\left(\frac{t}{2}\right) \omega_{p}(t, f)^{q} \frac{d t}{t}\right)^{1 / q}
\end{aligned}
$$

All together this results in

$$
\left(\sum_{j=0}^{\infty} \varrho^{q}\left(2^{-j}\right)\left\|f-S^{j} f \mid L_{p}\right\|^{q}\right)^{1 / q} \leq C\left(\left\|f \mid L_{p}\right\|^{q}+\int_{0}^{1} \varrho^{q}(t) \omega_{p}(t, f)^{q} \frac{d t}{t}\right)^{1 / q}
$$

choosing $N$ large enough.

Step 2. To prove the remaining inequality we employ some maximal function technique, cf. Appendix B. Following Triebel [30, formula 2.5.11/(6),(7)] we derive

$$
\begin{aligned}
\sup _{|h|<2^{-\ell}}\left|\Delta_{h}^{1} f(x)\right| & \leq \sup _{|h|<2^{-\ell}}\left|\Delta_{h^{1}}^{1} S^{\ell} f(x)\right|+\sup _{|h|<2^{-\ell}}\left|\Delta_{h}^{1}\left(f-S^{\ell} f\right)(x)\right| \\
& \leq c\left(\sum_{j=0}^{\ell} 2^{-\ell+j}\left|\varphi_{j}^{*, a} f(x)\right|+\left|\left(f-S^{\ell} f\right)(x)\right|\right)
\end{aligned}
$$


where $c$ is independent of $f, \ell, j$ and $x$. Hence, making use of a corresponding maximal inequality and the triangle inequality in $\ell_{q}$ we find

$$
\begin{aligned}
\left(\int_{0}^{1}\left(\varrho(t) \omega_{p}(t, f)\right)^{q} \frac{d t}{t}\right)^{1 / q} \leq & \left(\sum_{\ell=0}^{\infty}\left(\varrho\left(2^{-\ell-1}\right) \sup _{|h|<2^{-\ell}}\left\|\Delta_{h}^{1} f \mid L_{p}\right\|\right)^{q}\right)^{1 / q} \\
\leq c\left\{\sum_{\ell=0}^{\infty} \varrho\left(2^{-\ell}\right)^{q}\right. & \left(\sum_{m=0}^{\ell} 2^{-m}\left\|S_{\ell-m} f \mid L_{p}\right\|\right)^{1 / q} \\
& \left.+\left(\sum_{\ell=0}^{\infty} \varrho\left(2^{-\ell}\right)^{q}\left\|f-S^{\ell} f \mid L_{p}\right\|^{q}\right)^{1 / q}\right\} .
\end{aligned}
$$

Now

$$
\begin{aligned}
\sum_{\ell=0}^{\infty} \varrho\left(2^{-\ell}\right)^{q}( & \left.\sum_{m=0}^{\ell} 2^{-m}\left\|S_{\ell-m} f \mid L_{p}\right\|\right)^{1 / q} \\
& \leq \sum_{m=0}^{\infty} 2^{-m}\left(\sum_{\ell=m}^{\infty} \varrho\left(2^{-\ell}\right)^{q}\left\|S_{\ell-m} f \mid L_{p}\right\|^{q}\right)^{1 / q} \\
& \leq c \sum_{m=0}^{\infty} 2^{-m} M 2^{m \alpha}\left(\sum_{\ell=m}^{\infty} \varrho^{q}\left(2^{m-\ell}\right)^{q}\left\|S_{\ell-m} f \mid L_{p}\right\|^{q}\right)^{1 / q} \\
& \leq c\left(\left\|f\left|L_{p}\left\|^{q}+\sum_{\ell=0}^{\infty} \varrho^{q}\left(2^{-\ell}\right)\right\| f-S^{\ell} f\right| L_{p}\right\|^{q}\right)^{1 / q},
\end{aligned}
$$

where we used (9.2) and $0 \leq \alpha<1$. This proves the desired assertion.

Remark 27 As a consequence of this characterization we obtain the monotonicity of $B_{p, q}^{\varrho}$ with respect to $q$.

Remark 28 The conditions on $\varrho$ are not very restrictive. It is mainly an upper bound near zero given by $1 / t$ (put $v=1 / t$ in the admissibility condition (9.2)). Examples satisfying the requirements on $\varrho$ are $\varrho(t)=t^{-\alpha}$, $0 \leq \alpha<1, \varrho(t)=|\ln t|^{\alpha}, \alpha>0$, and $\varrho(t)=\ln ^{\alpha}|\ln t|, \alpha>0$ for small $t$.

Lemma 29 Suppose @ satisfies (9.2). Then the following assertions are equivalent:

(i) $\quad C_{\infty}^{\varrho} \hookrightarrow\left\{f \in C: \sup _{j=1,2, \ldots} j\left\|S_{j} f \mid L_{\infty}\right\|<\infty\right\} ;$

(ii) $\quad C_{\infty}^{\varrho} \hookrightarrow C_{\infty}^{|\ln |}$;

(iii) there exist constants $c_{1}$ and $c_{2}$ such that

$$
|\ln t| \leq c_{1} \varrho(t) \quad \text { for all } \quad t<c_{2} \leq \frac{1}{2} .
$$


Proof. The equivalence of (ii) and (iii) is obvious. (iii) implies (i) by Proposition 26. It remains to prove that (i) implies (iii). Let $\sigma \in \mathcal{S}$ be a function such that supp $\mathcal{F} \sigma$ is supported in a ball of radius $\frac{1}{4}$ and center $(7 / 4,0, \ldots, 0)$. Further, we may assume $\sup _{x \in \mathbb{R}^{n}}|\sigma(x)|=\sigma(0)=1$. Then we investigate functions of the type:

$$
f(x)=\sum_{j=1}^{\infty} \alpha_{j} \sigma\left(2^{j-1} x\right) .
$$

The advantage of this construction consists in $S_{0} f \equiv 0, S_{j} f(x)=\alpha_{j} \sigma\left(2^{j-1} x\right)$ and $\left\|S_{j} f\left|L_{\infty} \|=\right| \alpha_{j} \mid\right.$ for all $j \geq 1$. By Proposition 26$\| f \mid C_{\infty}^{\varrho} \|<\infty$ if

$$
\sum_{j=1}^{\infty}\left|\alpha_{j}\right|<\infty \quad \text { and } \quad \sup _{j=0,1, \ldots} \varrho\left(2^{-j}\right)\left|\sum_{k=j+1}^{\infty} \alpha_{k}\right|<\infty .
$$

Now we assume that (ii) is not true. Then there exists a sequence of points $t_{j}$ tending to zero and satisfying

$$
j^{3} \varrho\left(t_{j}\right)<\left|\ln t_{j}\right| .
$$

By monotonicity of $\varrho$ we may assume $t_{j}=2^{-\ell(j)}$, where $\ell(j)$ denotes an appropriate sequence of natural numbers. In view of $\varrho(t) \geq 1$ this implies $\ell(j) \geq j$. Choosing $\alpha_{\ell(j)}=1 /\left(j^{2} \varrho\left(2^{-\ell(j)}\right)\right)$ and $\alpha_{k}=0$ if $k \neq \ell(j)$ for all $j$, then the corresponding $f$ belongs to $C_{\infty}^{\varrho}$ but $\alpha_{\ell(j)} \ell(j)>j$. Hence (i) implies (iii).

Lemma 30 (i) Let $1 \leq q<\infty$. Then $C_{q}^{\ln } \hookrightarrow C_{D}=C_{1} \hookrightarrow C_{\infty}^{\ln } \cap B_{\infty, 1}^{0}$.

(ii) We have $C_{q}^{\ln } \hookrightarrow B_{\infty, 1}^{0}$ if and only if $q<\infty$.

Proof. Step 1. We deal with the second part of the chain of embeddings (i). We employ Proposition 26 and the following monotonicity property. To this end let $\left\|f-S^{j} f \mid L_{\infty}\right\|=\alpha_{j}$. Suppose $k<j$ and $\alpha_{k}<\alpha_{j}$. Then

$$
\begin{aligned}
\alpha_{j} & \leq\left\|f-S^{k} f\left|L_{\infty}\|+\| S^{j}\left(S^{k} f-f\right)\right| L_{\infty}\right\| \\
& \leq\left(1+\left\|\mathcal{F}^{-1} \varphi_{1}\left|L_{1}\|+\| \mathcal{F}^{-1} \varphi_{0}\right| L_{1}\right\|\right) \alpha_{k} .
\end{aligned}
$$

Hence, the sequence $\alpha_{j}$ is essentially monotone. Elementary analysis yields that $2^{j} \alpha_{2^{j}} \rightarrow 0$ for $j \rightarrow \infty$. Consequently, the sequence $(\ln j) \alpha_{j}$ is bounded. The embedding $C_{D} \hookrightarrow B_{\infty, 1}^{0}$ follows simply by Proposition 26 and the triangle inequality. 
Step 2. To prove the first part it is enough to apply Proposition 26 together with Hölder's inequality.

Step 3. We prove (ii). The function

$$
f(x)=\sum_{k=1}^{\infty} k^{-1} \varrho\left(2^{k-1}\left(x-x^{k}\right)\right)
$$

does not belong to $B_{\infty, 1}^{0}$. To guarantee $f \in C_{\infty}^{\ln }$ we choose $x^{k}=\left(2^{2 k}, 0, \ldots, 0\right)$. Then the assertion follows from

$$
\begin{aligned}
k\left|\sum_{j=k}^{\infty} S_{k} f(x)\right| & =k\left|\sum_{j=k}^{\infty} j^{-1} \varrho\left(2^{j-1}\left(x-x^{j}\right)\right)\right| \\
& \leq c_{M} k \sum_{j=k}^{\infty} j^{-1}\left(1+2^{j-1}\left|x-x^{j}\right|\right)^{-M} \\
& \leq c_{M} \sum_{j=1}^{\infty}\left(1+2^{j-1}\left|x-x^{j}\right|\right)^{-M} \\
& \leq C<\infty
\end{aligned}
$$

\section{References}

[1] Bourdaud, G.: Fonctions qui operent sur les espaces de Besov et de Triebel. Ann. Inst. H. Poincaré Anal. Non Linéaire 10 (1993), 413-422.

[2] Bourdaud, G. And Meyer, Y.: Fonctions qui operent sur les espaces de Sobolev. J. Funct. Anal. 97 (1991), 351-360.

[3] Caffarelli, L.A. And Kenig, C.E.: Gradient estimates for variable coefficient parabolic equations and singular perturbation problems. Amer. J. Math. 120(2), (1998), 391-439.

[4] Franke, J.: On the spaces $F_{p, q}^{s}$ of Triebel-Lizorkin type: Pointwise multipliers and spaces on domains. Math. Nachr. 125 (1986), 29-68.

[5] Frazier, M. and Jawerth, B.: A discrete transform and decomposition of distribution spaces. J. Funct. Anal. 93 (1990), 34-170.

[6] Frazier, M., Jawerth, B. And Weiss, G.: Littlewood-Paley theory and the study of function spaces. CBMS Reg. Conf. Ser. Math. 79, 1991.

[7] Goldberg, D.: A local version of real Hardy spaces. Duke Math. J. 46 (1979), 27-42.

[8] Gol'dman, M.L.: Description of trace classes of functions in generalized Hölder classes. Dokl. Akad. Nauk. SSSR 231 (1976), 525-528.

[9] Gol'dman, M.L.: Imbedding theorems for anisotropic Nikol'skij-Besov spaces with generalized modulus of smoothness. Proc. Steklov Inst. Math. 170 (1984), 86-104. 
[10] Gol'dman, M.L.: Imbedding theorems for generalized Nikol'skij-Besov spaces into Lorentz classes. Proc. Steklov Inst. Math. 172 (1985), 128-139.

[11] Grueter, M. and Widman, K.O.: The Green function for uniformly elliptic equations. Manuscripta Math. 37 (1982), 303-342

[12] Janson, S.: On functions with conditions on the mean oscillation. Ark. Mat. 14 (1976), 189-196.

[13] Johnsen, J.: Pointwise multiplication of Besov and Triebel-Lizorkin spaces. Math. Nachr. 175 (1995), 85-133.

[14] Marschall, J.: Remarks on Triebel spaces.Studia Math. 87 (1987), 79-92.

[15] Marschall, J.: On the boundedness and compactness of nonregular pseudodifferential operators. Math. Nachr. 175 (1995), 231-262.

[16] Marschall, J.: Remarks on nonregular pseudo-differential operators. Z. Anal. Anwendungen 15 (1996), 109-148.

[17] MaZ'ya, V.G. And Shaposhnikowa, T.O.: Theory of multipliers in spaces of differentiable functions. Pitman, Boston, 1985.

[18] Nikol'skiJ, S.M.: Approximation of functions of several variables and imbedding theorems. Springer, Berlin, 1975.

[19] Oberguggenberger, M.: Multiplication of distributions and applications to partial differential equations. Pitman Res. Notes Math. Ser. 259, Longman Scientific \& Technical, Harlow, 1992.

[20] Peetre, J.: New thoughts on Besov spaces. Duke Univ. Math. Series, Durham, 1976.

[21] Runst, T. And Sickel, W.: Sobolev spaces of fractional order, Nemytskij operators and nonlinear partial differential equations. De Gruyter, Berlin, 1996.

[22] Rychkov, V.S.: On a theorem of Bui, Paluszynski and Taibleson. Proc. Steklov Inst. Math. 277 (1999), 286-298.

[23] Seeger, A.: Remarks on singular convolution operators. Studia Math. 97 (1990), 91-114.

[24] Sickel, W.: On pointwise multipliers for $F_{p, q}^{s}$, the case $\sigma_{p, q}<s<n / p$. Ann. Mat. Pura. Appl. 176 (1999), 209-250.

[25] Sickel, W. And Smirnow, I.: Localization properties of Besov spaces and its associated multiplier spaces. Jenaer Schriften Math/Inf 21/99, Jena, 1999.

[26] Sickel, W. AND Triebel, H.: Hölder inequalities and sharp embeddings in function spaces of $B_{p, q}^{s}$ and $F_{p, q}^{s}$ type. Z. Anal. Anwendungen 14 (1995), $105-140$.

[27] Stegenga, D.A.: Bounded Toeplitz operators on $H^{1}$ and applications of duality between $H^{1}$ and the functions of bounded mean oscillations. Amer. J. Math. 98 (1976), 573-589.

[28] Strichartz, R.S.: Multipliers on fractional Sobolev spaces. J. Math. and Mech. 16 (1967), 1031-1060. 
[29] TAylor, M.E.: Pseudodifferential Operators and nonlinear PDE. Birkhäuser, Boston, 1991.

[30] Triebel, H.: Theory of function spaces. Birkhäuser, Basel, 1983.

[31] Triebel, H.: Theory of function spaces (russian). Mir, Moscow, 1986.

[32] Yamazaki, M.: A quasi-homogeneous version of paradifferential operators I: Boundedness on spaces of Besov type. J. Fac. Sci. Univ. Tokyo Sect. IA Math. 33 (1986), 131-174. A quasi-homogeneous version of paradifferential operators II: A Symbolic calculus. Ibidem 33 (1986), 311-345.

Recibido: 19 de septiembre de 2000

Revisado: 22 de marzo de 2001

Herbert Koch

Fachbereich Mathematik

Universität Dortmund

44221 Dortmund, Germany koch@math.uni-dortmund.de

Winfried Sickel

Mathematisches Institut

Friedrich-Schiller-Universität Jena

07740 Jena, Germany sickel@minet.uni-jena.de 